\title{
NATIONAL ACADEMY OF SCIENCES
}

\author{
July 1, 1967 \\ OFFICERS
}

President-Frederick Seitz

Vice President-G. B. Kistiakowsky

Home Secretary-Merle A. Tuve

Foreign Secretary-Harrison Brown

Treasurer-E. R: Piore

Executive Officer

John S. Coleman

\author{
Term expires \\ June 30, 1971 \\ June 30, 1969 \\ June 30, 1971 \\ June 30, 1970 \\ June 30, 1968 \\ Business Manager \\ G. D. Meid
}

\section{COUNCIL}

$\begin{array}{ll}\text { Bode, H. W. } & (\mathbf{1 9 7 0 )} \\ \text { *Brown, Harrison } & (\mathbf{1 9 7 0 )} \\ \text { Carter, H. E. } & (1969) \\ \text { Eggan, Fred } & (\mathbf{1 9 7 0 )} \\ \text { Esau, Katherine } & (\mathbf{1 9 6 9 )} \\ \text { Fenn, Wallace O. } & (\mathbf{1 9 6 9 )} \\ \text { Flory, Paul J. } & (\mathbf{1 9 7 0 )} \\ \text { Greenstein, Jesse L. } & (\mathbf{1 9 6 9 )} \\ \text { Hendricks, Sterling B. } & (\mathbf{1 9 6 8 )}\end{array}$

\section{SECTIONS}

The Academy is divided into the following Sections, to which members are assigned at their own choice:
(1) Mathematics
(2) Astronomy
(3) Physics
(4) Engineering
(5) Chemistry
(6) Geology
(7) Botany
(8) Zoology and Anatomy

*Kistiakowsky, G. B.

*MacLeod, Colin M.

McShane, E. J.

*Piore, E. R.

*Rubey, William W.

*Seitz, Frederick

*Tuve, Merle A.

Weinberg, Alvin
(1969)

(1968)

(1970)

In the list of members below, the number in parentheses, following year of election, indicates the Section to which the member belongs.

\section{CLASSES}

The members of Sections are grouped in the following Classes:

(1) Physical and Mathematical Sciences (Sections 1, 2, 3, 5, 6, 13)

(2) Biological and Behavioral Sciences (Sections 7, 8, 9, 10, 11, 12, 14)

(3) Engineering and Applied Sciences (Sections 4, 15, 16)

With the approval of the Home Secretary any member may at his request be assigned to a Class different from that to which his Section as a whole is assigned. Only in such cases is the Class shown in the list of members below.

* Members of the Executive Committee of the Council of the Academy. 


\section{MEMBERS}

Abbot, Charles Greeley, 1915 (2), Smithsonian Institution, Washington, D. C. 20560

Abelson, Philip Hauge, 1959 (6), Geophysical Laboratory, Carnegie Institution of Washington, 2801 Upton Street, N. W., Washington, D. C. 20008

Adams, Leason Heberling, 1943 (13), 8700 Rayburn Road, Bethesda, Maryland 20034

Adams, Roger, 1929 (5), Department of Chemistry and Chemical Engineering, 266 East Chemistry Building, University of Illinois, Urbana, Illinois 61801

Ahlfors, Lars Valerian, 1953 (1), Department of Mathematics, Harvard University, 2 Divinity Avenue, Cambridge, Massachusetts 02138

Albert, Abraham Adrian, 1943 (1), 111 Eckhart Hall, University of Chicago, 1118 East 58th Street, Chicago, Illinois 60637

Alberty, Robert Arnold, 1965 (5), School of Science, Massachusetts Institute of Technology, 77 Massachusetts Avenue, Cambridge, Massachusetts 02139

Albright, William Foxwell, 1955 (11), Department of Near Eastern Studies, Johns Hopkins University, Baltimore, Maryland 21218

Alexander, James Waddell, 1930 (1), 29 Cleveland Lane, Princeton, New Jersey 08540

Aller, Lawrence Hugh, 1962 (2), Department of Astronomy, University of California, Los Angeles, California 90024

Alvarez, Luis Walter, 1947 (3), Lawrence Radiation Laboratory, University of California, Berkeley, California 94720

Anderson, Carl David, 1938 (3), California Institute of Technology, Pasadena, California 91109

Anderson, Charles Alfred, 1957 (6), United States Geological Survey, 345 Middlefield Road, Menlo Park, California 94025

Anderson, Edgar, 1954 (7), Missouri Botanical Garden, 2315 Tower Grove Avenue, St. Louis, Missouri 63110

Anderson, Herbert Lawrence, 1960 (3), The Enrico Fermi Institute for Nuclear Studies, University of Chicago, Chicago, Illinois 60637

Anderson, Philip Warren, 1967 (3), Room 1D-246, Bell Telephone Laboratories, Incorporated, Murray Hill, New Jersey 07971

Anderson, Thomas Foxen, 1964 (7), The Institute for Cancer Research, 7701 Burholme Avenue, Philadelphia, Pennsylvania 19111

Anfinsen, Christian Boehmer, 1963 (14), Laboratory of Chemical Biology, National Institute of Arthritis and Metabolic Diseases, National Institutes of Health, Bethesda, Maryland 20014

Arnold, James Richard, 1964 (13), Department of Chemistry, University of California, San Diego, La Jolla, California 92037

Arnold, William Archibald, 1962 (7), Biology Division, Oak Ridge National Laboratory, P. O. Box Y, Oak Ridge, Tennessee 37831

Arnon, Daniel Israel, 1961 (7), Department of Cell Physiology, Hilgard Hall, University of California, Berkeley, California 94720 
Astin, Allen Varley, 1960 (4), National Bureau of Standards, Washington, D. C. 20234

Astwood, Edwin Bennett, 1957 (9), New England Medical Center Hospitals, Harrison Avenue and Bennet Street, Boston, Massachusetts 02111

Aub, Joseph Charles, 1957 (9), 233 Prospect Street, Belmont, Massachusetts 02178

Babcock, Horace Welcome, 1954 (2), Mount Wilson and Palomar Observatories, 813 Santa Barbara Street, Pasadena, California 91106

Bacher, Robert Fox, 1947 (3), California Institute of Technology, Pasadena, California 91109

Badger, Richard McLean, 1952 (5), Gates and Crellin Laboratories of Chemistry, California Institute of Technology, Pasadena, California 91109

Bailey, Percival, 1953 (10), 731 Lincoln Street, Evanston, Illinois 60201

Bain, Edgar Collins, 1954 (4), 434 Maple Lane, Edgeworth, Sewickley, Pennsylvania 15143

Bainbridge, Kenneth Tompkins, 1946 (3), Department of Physics, Harvard University, Cambridge, Massachusetts 02138

Baker, James Gilbert, 1965 (2), Harvard College Observatory, Cambridge, Massachusetts 02138

Baker, William Oliver, 1961 (5), Bell Telephone Laboratories, Incorporated, Murray Hill, New Jersey 07971

Ball, Eric Glendinning, 1948 (14), Department of Biological Chemistry, Harvard Medical Sichool, 25 Shattuck Street, Boston, Massachusetts 02115

Bard, Philip, 1944 (9), School of Medicine, Johns Hopkins University, 725 North Wolfe Street, Baltimore, Maryland 21205

Bardeen, Johṇ, 1954 (3), Department of Physics, University of Illinois, Urbana, Illinois 61801

Barghoorn, Elso Sterrenberg, 1967 (7), The Biological Laboratories, Harvard University, 16 Divinity Avenue, Cambridge, Massachusetts 02138

Barker, Horace Albert, 1953 (7), Department of Biochemistry, Biochemistry Building, University of California, Berkeley, California 94720

Barrett, Charles Sanborn, 1967 (4), Institute for the Study of Metals, University of Chicago, Chicago, Illinois 60637

Bartlett, Paul Doughty, 1947 (5), Department of Chemistry, Harvard University, 12 Oxford Street, Cambridge, Massachusetts 02138

Beach, Frank Ambrose, 1949 (12), Department of Psychology, University of California, Berkeley, California 94720

Beadle, George Wells, 1944 (7), University of Chicago, 5801 Ellis Avenue, Chicago, Illinois 60637

Beams, Jesse Wakefield, 1943 (3), Physics Laboratory, McCormick Road, University of Virginia, Charlottesville, Virginia 22903

Benedict, Manson, 1956 (4), Room 24-109, Department of Nuclear Engineering, Massachusetts Institute of Technology, Cambridge, Massachusetts 02139

Benioff, Victor Hugo, 1953 (13), Comptche-Ukiah Road, Mendocino, California 95460

Benzer, Seymour, 1961 (7), (On leave from Purdue University): Division of Biology, California Institute of Technology, Pasadena, California 91109

Berg, Paul, 1966 (14), Department of Biochemistry, Stanford University School of Medicine, Palo Alto, California 94304 
Bers, Lipman, 1964 (1), Department of Mathematics, Columbia University, New York, New York 10027

Bethe, Hans Albrecht, 1944 (3), Laboratory of Nuclear Studies, Cornell University, Ithaca, New York 14850

Bigeleisen, Jacob, 1966 (5), Department of Chemistry, Brookhaven National Laboratory, Upton, New York 11973

Bigelow, Henry Bryant, 1931 (8), Museum of Comparative Zoology at Harvard College, Oxford Street, Cambridge, Massachusetts 02138

Bing, R. H., 1965 (1), Department of Mathematics, University of Wisconsin, Madison, Wisconsin 53706. At Institute for Advanced Study, Princeton, SeptemberDecember 1967; University of California, Los Angeles, January-March 1968

Birch, Albert Francis, 1950 (6), Department of Geological Sciences, Harvard University, Cambridge, Massachusetts 02138

Birge, Raymond Thayer, 1932 (3), Department of Physics, University of California, Berkeley, California 94720

Bishop, George Holman, 1967 (9), Department of Neurology, School of Medicine, Washington University, St. Louis, Missouri 63110

Bisplinghoff, Raymond Lewis, 1967 (4), Room 33-207, Department of Aeronautics and Astronautics, Massachusetts Institute of Technology, Cambridge, Massachusetts 02139

Bjerknes, Jacob, 1947 (13), Department of Meteorology, University of California, Los Angeles, California 90024

Blackwelder, Eliot, 1936 (6), 2172 The Alameda, Redwood City, California 94061

Blackwell, David Harold, 1965 (1), Department of Statistics, University of California, Berkeley, California 94720

Bleakney, Walker, 1959 (3), Palmer Physical Laboratory, Princeton University, P. O. Box 708, Princeton, New Jersey 08540

Blinks, Lawrence Rogers, 1955 (7), Hopkins Marine Station of Stanford University, Pacific Grove, California 93950

Bloch, Felix, 1948 (3), Department of Physics, Stanford University, Stanford, California 94305

Bloch, Konrad Emil, 1956 (14), Department of Chemistry, Harvard University 12 Oxford Street, Cambridge, Massachusetts 02138

Bloembergen, Nicolaas, 1960 (3), Pierce Hall, Harvard University, Cambridge, Massachusetts 02138

Blomquist, Alfred Theodore, 1960 (5), Department of Chemistry, Cornell University, Ithaca, New York 14850

Bloom, William, 1954 (8), University of Chicago, 5640 South Ellis Avenue, Chicago, Illinois 60637

Bochner, Salomon, 1950 (1), Department of Mathematics, Princeton University, P. O. Box 708, Princeton, New Jersey 08540

Bode, Hendrik Wade, 1957 (4), Bell Telephone Laboratories, Incorporated, Whippany, New Jersey 07981

Bodenstein, Dietrich H. F. A., 1958 (8), Department of Biology, University of Virginia, Charlottesville, Virginia 22903

Bodian, David, 1958 (8), Department of Anatomy, School of Medicine, Johns Hopkins University, 725 North Wolfe Street, Baltimore, Maryland 21205 
Boekelheide, Virgil Carl, 1962 (5), Department of Chemistry, University of Oregon, Eugene, Oregon 97403

Bolton, Elmer K., 1946 (5), 2310 West 11th Street, Wilmington, Delaware 19805

Bonner, James Frederick, 1950 (7), Division of Biology, California Institute of Technology, Pasadena, California 91109

Booker, Henry George, 1960 (13), Department of Applied Electrophysics, University of California, San Diego, La Jolla, California 92037

Boring, Edwin Garrigues, 1932 (12), William James Hall, Harvard University, 33 Kirkland Street, Cambridge, Massachusetts 02138

Borthwick, Harry Alfred, 1961 (7), Plant Industry Station, United States Department of Agriculture, Beltsville, Maryland 20705

Bott, Raoul, 1964 (1), Department of Mathematics, Harvard University, 2 Divinity Avenue, Cambridge, Massachusetts 02138

Bowen, Ira Sprague, 1936 (2), Mount Wilson and Palomar Observatories, 813 Santa Barbara Street, Pasadena, California 91106

Bradbury, Norris Edwin, 1951 (3), Los Alamos Scientific Laboratory, P. O. Box 1663, Los Alamos, New Mexico 87544

Bradley, Wilmot Hyde, 1946 (6), United States Geological Survey, Washington, D. C. 20242

Braidwood, Robert John, 1964 (11), The Oriental Institute, University of Chicago, Chicago, Illinois 60637

Bramlette, Milton Nunn, 1954 (6), Scripps Institution of Oceanography, La Jolla, California 92037

Brattain, Walter Houser, 1959 (3), Whitman College, Walla Walla, Washington 99362

Brauer, Richard Dagobert, 1955 (1), Department of Mathematics, Harvard University, 2 Divinity Avenue, Cambridge, Massachusetts 02138

Braun, Armin Charles, 1960 (7), Rockefeller University, New York, New York 10021

Breit, Gregory, 1939 (3), Sloane Physics Laboratory, Yale University, P. O. Box 2014, New Haven, Connecticut 06520

Breslow, Ronald, 1966 (5), Department of Chemistry, Columbia University, New York, New York 10027

Brewer, Leo, 1959 (5), Department of Chemistry, University of California, Berkeley, California 94720

Briggs, Robert William, 1962 (8), Department of Zoology, Indiana University, Bloomington, Indiana 47401

Brillouin, Leon, 1953 (3), 88 Central Park West, New York, New York 10023

Brink, Frank, Jr., 1959 (9), Rockefeller University, New York, New York 10021

Brink, Royal Alexander, 1947 (7), Department of Genetics, University of Wisconsin, Madison, Wisconsin 53706

Brode, Robert Bigham, 1949 (3), Department of Physics, University of California, Berkeley, California 94720

Brode, Wallace Reed, 1954 (5), 3900 Connecticut Avenue, N. W., Washington, D. C. 20008

Brodie, Bernard Beryl, 1966 (9), Laboratory of Chemical Pharmacology, National Heart Institute, National Institutes of Health, Bethesda, Maryland 20014 
Bronk, Detlev Wulf, 1939 (9), Rockefeller University, New York, New York 10021

Brooks, Harvey, 1962 (4), 217 Pierce Hall, Harvard University, Cambridge, Massachusetts 02138

Brown, Harrison Scott, 1955 (13), Division of Geological Sciences, California Institute' of Technology, Pasadena, California 91109

Brown, Herbert Charles, 1957 (5), Department of Chemistry, Purdue University, Lafayette, Indiana 47907

Buchanan, John Machlin, 1962 (14), Room 16-619, Department of Biology, Massachusetts Institute of Technology, Cambridge, Massachusetts 02139

Büchi, George H., 1965 (5), Room 6-335, Department of Chemistry, Massachusetts Institute of Technology, Cambridge, Massachusetts 02139

Buddington, Arthur Francis, 1943 (6), Department of Geology, Princeton University, Princeton, New Jersey 08540

Buerger, Martin Julian, 1953 (6), Room 54-814, Department of Geology and Geophysics, Massachusetts Institute of Technology, Cambridge, Massachusetts 02139

Bullock, Theodore Holmes, 1963 (8), Department of Neurosciences, School of Medicine, University of California, San Diego, La Jolla, California 92037

Burkholder, Paul Rufus, 1949 (7), Lamont Geological Observatory of Columbia University, Torrey Cliff, Palisades, New York 10964

Burns, Robert Kyle, 1955 (8), Department of Biology, Bridgewater College, Bridgewater, Virginia 22812

Burris, Robert Harza, 1961 (7), Department of Biochemistry, University of Wisconsin, Madison, Wisconsin 53706

Bush, Vannevar, 1934 (4), Room 10-211, Massachusetts Institute of Technology, Cambridge, Massachusetts 02139

Byerly, Perry, 1946 (13), Seismographic Stations, University of California, Berkeley, California 94720

Byers, Horace Robert, 1952 (13), College of Geosciences, Texas A \& M University, College Station, Texas 77843

Cairns, Theodore L., 1966 (5), Central Research Department, Experimental Station, E. I. du Pont de Nemours \& Company, Incorporated, Wilmington, Delaware 19898

Calvin, Melvin, 1954 (5), Laboratory of Chemical Biodynamics, University of California, Berkeley, California 94720

Cannon, Paul Roberts, 1946 (10), Box 56, Route 2, Yorkville, Illinois 60560

Carmichael, Leonard, 1943 (12), National Geographic Society, 17th and M Streets, N. W., Washington, D. C. 20036

Carrier, George Francis, 1967 (4), Division of Engineering and Applied Physics, Pierce Hall, Harvard University, Cambridge, Massachusetts 02138

Carter, Herbert Edmund, 1953 (14), 112b English Building, University of Illinois, Urbana, Illinois 61801

Castle, William Bosworth, 1939 (10), Thorndike Memorial Laboratory, Boston City Hospital, Boston, Massachusetts 02118

Chamberlain, Joseph Wyan, 1965 (13), Kitt Peak National Observatory, 950 North Cherry Avenue, P. O. Box 4130, Tucson, Arizona 85717

Chamberlain, Owen, 1960 (3), Department of Physics, University of California, Berkeley, California 94720 
Chance, Britton, 1954 (14), Johnson Research Foundation, Department of Biophysics and Physical Biochemistry, University of Pennsylvania, Philadelphia, Pennsylvania 19104

Chandler, William Henry, 1943 (7), 3012 Hillegass Avenue, Berkeley, California 94705

Chandrasekhar, Subrahmanyan, 1955 (2), Laboratory for Astrophysics and Space Research, 933 East 56th Street, Chicago, Illinois 60637

Chaney, Ralph Works, 1947 (6), Department of Paleontology, University of California, Berkeley, California 94720

Chargaff, Erwin, 1965 (14), Columbia University College of Physicians and Surgeons, 630 West 168th Street, New York, New York 10032

Charney, Jule Gregory, 1964 (13), Department of Meteorology, Massachusetts Institute of Technology, Cambridge, Massachusetts 02139

Chern, Shiing-shen, 1961 (1), Department of Mathematics, University of California, Berkeley, California 94720

Chew, Geoffrey Foucar, 1962 (3), Department of Physics, University of California, Berkeley, California 94720

Chipman, John, 1955 (4), Room 16-402, Department of Metallurgy, Massachusetts Institute of Technology, Cambridge, Massachusetts 02139

Christy, Robert Frederick, 1965 (3), Department of Physics, California Institute of Technology, Pasadena, California 91109

Clarke, Hans Thacher, 1942 (14), 19 Ware Street, Cambridge, Massachusetts 02138

Clausen, Jens Christian, 1959 (7), Department of Plant Biology, Carnegie Institution of Washington, Stanford, California 94305

Cleland, Ralph Erskine, 1942 (7), Department of Botany, Indiana University, Bloomington, Indiana 47401

Clemence, Gerald Maurice, 1952 (2), Yale University Observatory, 2023 Yale Station, New Haven, Connecticut 06520

Cleveland, Lemuel Roscoe, 1952 (8), Route 5, Union, Mississippi 39365

Cloos, Ernst, 1950 (6), Department of Geology, Johns Hopkins University, Baltimore, Maryland 21218

Cloud, Preston E., Jr., 1961 (6), Department of Geology, University of California, Los Angeles, California 90024

Coggeshall, Lowell Thelwell, 1949 (10), Route 3, Foley, Alabama 36535

Cohen, Paul Joseph, 1967 (1), Department of Mathematics, Stanford University, Stanford, California 94305

Cohen, Seymour Stanley, 1967 (14), Department of Therapeutic Research, University of Pennsylvania School of Medicine, Philadelphia, Pennsylvania 19104

Colbert, Edwin Harris, 1957 (8), American Museum of Natural History, Central Park West at 79th Street, New York, New York 10024

Cole, Kenneth Stewart, 1956 (9), Laboratory of Biophysics, National Institute of Neurological Diseases and Blindness, National Institutes of Health, Bethesda, Maryland 20014

Comroe, Julius Hiram, Jr., 1961 (9), Cardiovascular Research Institute, University of California Medical Center, San Francisco, California 94122

Conant, James Bryant, 1929 (5), Manhattan House, 200 East 66th Street, New York, New York 10021 
Condon, Edward Uhler, 1944 (3), 761 Cascade Avenue, Boulder, Colorado 80302

Connick, Robert Elwell, 1963 (5), College of Chemistry, 420 Latimer Hall, University of California, Berkeley, California 94720

Coon, Carleton Stevens, 1955 (11), 207 Concord Street, Gloucester, Massachusetts 01930

Coons, Albert Hewett, 1962 (10), Department of Bacteriology and Immunology, Harvard Medical School, 25 Shattuck Street, Boston, Massachusetts 02115

Corey, Elias James, 1966 (5), Department of Chemistry, Harvard University, 12 Oxford Street, Cambridge, Massachusetts 02138

Cori, Carl Ferdinand, 1940 (14), Massachusetts General Hospital, Fruit Street, Boston, Massachusetts 02114

Corner, George Washington, 1940 (8), American Philosophical Society, 104 South Fifth Street, Philadelphia, Pennsylvania 19106

Cotton, Frank Albert, 1967 (5), Department of Chemistry, Massachusetts Institute of Technology, Cambridge, Massachusetts 02139

Couch, John Nathaniel, 1943 (7), University of North Carolina, Chapel Hill, North Carolina 27514

Courant, Richard, 1955 (1), Courant Institute of Mathematical Sciences, New York University, 251 Mercer Street, New York, New York 10012

Cournand, André Frederic, 1958 (9), Cardio-Pulmonary Laboratory (Columbia University Division), Bellevue Hospital, 462 First Avenue, New York, New York 10016

Craig, Lyman Creighton, 1950 (14), Rockefeller University, New York, New York 10021

Cram, Donald James, 1961 (5), Department of Chemistry, University of California, Los Angeles, California 90024

Crane, Horace Richard, 1966 (3), Randall Laboratory of Physics, University of Michigan, Ann Arbor, Michigan 48104

Crawford, Bryce, Jr., 1956 (5), Graduate School, University of Minnesota, Minneapolis, Minnesota 55455

Crow, James Franklin, 1961 (8), Department of Medical Genetics, University of Wisconsin, Madison, Wisconsin 53706

Curme, George Oliver, Jr., 1944 (4), Union Carbide Corporation, 270 Park Avenue, 35th Floor, New York, New York, 10017

Curtin, David Yarrow, 1964 (5), Department of Chemistry and Chemical Engineering, University of Illinois, Urbana, Illinois 61801

Dalldorf, Gilbert, 1955 (10), Oxford, Maryland 21654

Danforth, Charles Haskell, 1942 (8), Department of Anatomy, Stanford University, Stanford, California 94305

Daniels, Farrington, 1947 (5), Solar Energy Laboratory, University of Wisconsin, Madison, Wisconsin 53706

Darken, Lawrence Stamper, 1961 (4), United States Steel Corporation, Research Center, M.S.59, Monroeville, Pennsylvania 15146

Darlington, Philip Jackson, Jr., 1964 (8), Museum of Comparative Zoology at Harvard College, Oxford Street, Cambridge, Massachusetts 02138

Davidson, Norman Ralph, 1960 (5), Gates and Crellin Laboratories of Chemistry, California Institute of Technology, Pasadena, California 91109 
Davis, Bernard David, 1967 (10), Department of Bacteriology and Immunology, Harvard Medical School, 25 Shattuck Street, Boston, Massachusetts 02115

Davis, Hallowell, 1948 (9), Central Institute for the Deaf, 818 South Euclid, St. Louis, Missouri 63110

Davis, Kingsley, 1966 (11), International Population and Urban Research, 2234 Piedmont Avenue, Berkeley, California 94720

Delbrück, Max, 1949 (7), California Institute of Technology, Pasadena, California 91109

Den Hartog, Jacob Pieter, 1953 (4), Room 3-260, Department of Mechanical Engineering, Massachusetts Institute of Technology, Cambridge, Massachusetts 02139

Dennison, David Mathias, 1953 (3), Physics-Astronomy Building, University of Michigan, Ann Arbor, Michigan 48104

Dethier, Vincent Gaston, 1965 (8), Department of Biology, Princeton University, Princeton, New Jersey 08540

Deutsch, Martin, 1958 (3), Room 26-419, Laboratory for Nuclear Science, Massachusetts Institute of Technology, Cambridge, Massachusetts 02139

Dicke, Robert Henry, 1967 (3), Palmer Physical Laboratory, Princeton University, Princeton, New Jersey 08540

Dingle, John Holmes, 1958 (10), Department of Preventive Medicine, Wearn Research Building, University Hospitals, Cleveland, Ohio 44106

Djerassi, Carl, 1961 (5), Department of Chemistry, Stanford University, Stanford, California 94305

Dobzhansky, Theodosius, 1943 (8), Rockefeller University, New York, New York 10021

Doering, William von Eggers, 1961 (5), Yale University, 1901 A Yale Station, New Haven, Connecticut 06520

Doisy, Edward Adelbert, 1938 (14), St. Louis University School of Medicine, 1402 South Grand Boulevard, St. Louis, Missouri 63104

Doob, Joseph Leo, 1957 (1), Department of Mathematics, University of Illinois, Urbana, Illinois 61801

Doty, Paul Mead, 1957 (14), Department of Chemistry, Harvard University, 12 Oxford Street, Cambridge, Massachusetts 02138

Doudoroff, Michael, 1962 (14), Department of Bacteriology and Immunology, University of California, Berkeley, California 94720

Dragstedt, Lester Reynold, 1950 (10), Department of Surgery, University of Florida, Gainesville, Florida 32603

Draper, Charles Stark, 1957 (4), IL3-415, Instrumentation Laboratory, Massachusetts Institute of Technology, Cambridge, Massachusetts 02139

Drickamer, Harry George, 1965 (5), Department of Chemistry and Chemical Engineering, University of Illinois, Urbana, Illinois 61801

Dubos, René Jules, 1941 (10), Rockefeller University, New York, New York 10021

DuBridge, Lee Alvin, 1943 (3), California Institute of Technology, Pasadena, California 91109

Dulbecco, Renato, 1961 (10), The Salk Institute for Biological Studies, Box 1809, San Diego, California 92112 
DuMond, Jesse William Monroe, 1953 (3), Department of Physics, California Institute of Technology, Pasadena, California 91109

Dunbar, Carl Owen, 1944 (6), 1615 Santa Barbara Drive, Dunedin, Florida 33528

Dunn, Leslie Clarence, 1943 (8), 635 West 247th Street, New York, New York 10471

Dunning, John Ray, 1948 (3), School of Engineering and Applied Science, 510 Mudd Building, Columbia University, New York, New York 10027

du Vigneaud, Vincent, 1944 (14), Department of Chemistry, Cornell University, Ithaca, New York 14850

Dyson, Freeman John, 1964 (3), The Institute for Advanced Study, Princeton, New Jersey 08540

Eagle, Harry, 1963 (10), Department of Cell Biology, Albert Einstein College of Medicine, Bronx, New York 10461

Ebert, James David, 1967 (8), Department of Embryology, Carnegie Institution of Washington, Baltimore, Maryland 21210

Eckart, Carl, 1953 (13), University of California, San Diego, La Jolla, California 92037

Edgerton, Harold Eugene, 1964 (4), Department of Electrical Engineering, Massachusetts Institute of Technology, Cambridge, Massachusetts 02139

Edsall, John Tileston, 1951 (14), The Biological Laboratories, Harvard University, 16 Divinity Avenue, Cambridge, Massachusetts 02138

Eggan, Fred Russell, 1963 (11), Department of Anthropology, University of Chicago, 1126 East 59th Street, Chicago, Illinois 60637

Eilenberg, Samuel, 1959 (1), Department of Mathematics, Columbia University, New York, New York 10027

Elderfield, Robert Cooley, 1949 (5), Department of Chemistry, University of Michigan, Ann Arbor, Michigan 48104

Elsasser, Walter Maurice, 1957 (13), Department of Geology, Princeton University, Princeton, New Jersey 08540

Emerson, Alfred Edwards, 1962 (8), Huletts Landing, New York 12841

Emmett, Paul Hugh, 1955 (5), Department of Chemistry, Johns Hopkins University, Baltimore, Maryland 21218

Emmons, Howard Wilson, 1966 (4), Pierce Hall, Harvard University, Cambridge, Massachusetts 02138

Enders, John Franklin, 1953 (10), The Children's Hospital Medical Center, 300 Longwood Avenue, Boston, Massachusetts 02115

Esau, Katherine, 1957 (7), Department of Biological Sciences, University of California, Santa Barbara, California 93106

Estes, William Kaye, 1963 (12), Department of Psychology, Stanford University, Stanford, California 94305

Evans, Griffith Conrad, 1933 (1), Department of Mathematics, University of California, Berkeley, California 94720

Evans, Herbert McLean, 1927 (9), Anatomy Department, 4579 Life Sciences Building, University of California, Berkeley, California 94720

Ewing, William Maurice, 1948 (13), Lamont Geological Observatory of Columbia University, Torrey Cliff, Palisades, New York 10964 
Eyring, Henry, 1945 (5), Department of Chemistry, University of Utah, Salt Lake City, Utah 84112

Fairbank, William Martin, 1963 (3), Department of Physics, Stanford University, Stanford, California 94305

Feller, William, 1960 (1), Fine Hall, Princeton University, Princeton, New Jersey 08540

Fenn, Wallace Osgood, 1943 (9), School of Medicine and Dentistry, University of Rochester, 260 Crittenden Boulevard, Rochester, New York 14620

Ferry, John Douglass, 1959 (5), Department of Chemistry, University of Wisconsin, Madison, Wisconsin 53706

Feynman, Richard Phillips, 1954 (3), Norman Bridge Laboratory of Physics, California Institute of Technology, Pasadena, California 91109

Fieser, Louis Frederick, 1940 (5), Harvard University, Cambridge, Massachusetts 02138

Fisk, James Brown, 1954 (4), Bell Telephone Laboratories, Incorporated, Murray Hill, New Jersey 07971

Fitch, Val Logsdon, 1966 (3), Palmer Physical Laboratory, Princeton University, Princeton, New Jersey 08540

Fletcher, Harvey, 1935 (4), 272 Eyring Science Center, Brigham Young University, Provo, Utah 84601

Flexner, Louis Barkhouse, 1964 (8), School of Medicine, University of Pennsylvania, Philadelphia, Pennsylvania 19104

Flory, Paul John, 1953 (5), Department of Chemistry, Stanford University, Stanford, California 94305

Folkers, Karl August, 1948 (5), Stanford Research Institute, Menlo Park, California 94025

Foote, Paul Darwin, 1943 (4), 5144 Macomb Street, N. W., Washington, D. C. 20016

Forbush, Scott Ellsworth, 1962 (13), Department of Terrestrial Magnetism, Carnegie Institution of Washington, 5241 Broad Branch Road, N. W., Washington, D. C. 20015

Fowler, William Alfred, 1956 (3), W. K. Kellogg Radiation Laboratory, California Institute of Technology, Pasadena, California 91109

Francis, Thomas, Jr., 1948 (10), Department of Epidemiology, School of Public Health, University of Michigan, Ann Arbor, Michigan 48104

Fred, Edwin Broun, 1931 (7), University of Wisconsin, Madison, Wisconsin 53706

French, Charles Stacy, 1963 (7), Department of Plant Biology, Carnegie Institution of Washington, Stanford, California 94305

Friedman, Herbert, 1960 (2), United States Naval Research Laboratory (Code 7100), Washington, D. C. 20390

Friedmann, Herbert, 1962 (8), Los Angeles County Museum of Natural History, 900 Exposition Boulevard, Los Angeles, California 90007

Friedrichs, Kurt Otto, 1959 (1), Courant Institute of Mathematical Sciences, New York University, 251 Mercer Street, New York, New York 10012

Fruton, Joseph Stewart, 1952 (14), 350 Kline Biology Tower, 219 Prospect Street, New Haven, Connecticut 06520 
Fuoss, Raymond Matthew, 1951 (5), 57 Mill Rock Road, New Haven, Connecticut 06511

Fuson, Reynold Clayton, 1944 (5), Department of Chemistry, University of Nevada, Reno, Nevada 89507

Galambos, Robert, 1960 (12), Yale University, 333 Cedar Street, New Haven, Connecticut 06510

Gamow, George, 1953 (3), Department of Physics, University of Colorado, Boulder, Colorado 80304

Garner, Wendell Richard, 1965 (12), Department of Psychology, Yale University, New Haven, Connecticut 06510

Garrels, Robert Minard, 1962 (6), Department of Geology, Northwestern University, Evanston, Illinois 60201

Garwin, Richard Lawrence, 1966 (3), IBM Watson Laboratory, 612 West 115th Street, New York, New York 10025

Gates, Marshall DeMotte, Jr., 1958 (5), Department of Chemistry, University of Rochester, Rochester, New York 14627

Gell-Mann, Murray, 1960 (3), Department of Physics, California Institute of Technology, Pasadena, California 91109

Gerard, Ralph Waldo, 1955 (9), University of California, Irvine, California 92664

Giauque, William Francis, 1936 (5), Department of Chemistry, University of California, Berkeley, California 94720

Gibbs, William Francis, 1949 (4), One Broadway, New York, New York 10004

Gibson, James Jerome, 1967 (12), Graduate Psychological Laboratories, Cornell University, Ithaca, New York 14850

Giles, Norman Henry, 1966 (7), Department of Biology, Yale University, New Haven, Connecticut 06520

Gilliland, Edwin Richard, 1948 (4), Room 12-117, Department of Chemical Engineering, Massachusetts Institute of Technology, Cambridge, Massachusetts 02139

Gilluly, James, 1947 (6), 975 Estes Street, Lakewood, Colorado 80215

Gilman, Alfred, 1964 (9), Department of Pharmacology, Albert Einstein College of Medicine, 1300 Morris Park Avenue, Bronx, New York 10461

Gilman, Henry, 1945 (5), Department of Chemistry, Iowa State University, Ames, Iowa 50012

Ginzton, Edward Leonard, 1966 (4), Varian Associates, 611 Hansen Way, Palo Alto, California 94303

Glaser, Donald Arthur, 1962 (3), Department of Molecular Biology, University of California, Berkeley, California 94720

Glass, Hiram Bentley, 1959 (8), State University of New York, Stony Brook, New York 11790

Gleason, Andrew Mattei, 1966 (1), Department of Mathematics, Harvard University, 2 Divinity Avenue, Cambridge, Massachusetts 02138

Goddard, David Rockwell, 1950 (7), Provost's Office, 102 College Hall, University of Pennsylvania, Philadelphia, Pennsylvania 19104

Gödel, Kurt, 1955 (1), The Institute for Advanced Study, Princeton, New Jersey 08540 
Goebel, Walther Frederick, 1958 (10), Rockefeller University, New York, New York 10021

Goldberg, Leo, 1958 (2), Harvard College Observatory, 60 Garden Street, Cambridge, Massachusetts 02138

Goldberger, Marvin Leonard, 1963 (3), Palmer Physical Laboratory, Princeton University, Princeton, New Jersey 08540

Goldhaber, Maurice, 1958 (3), Director's Office, Brookhaven National Laboratory, Upton, New York 11973

Goodman, Louis Sanford, 1965 (9), Department of Pharmacology, University of Utah, College of Medicine, Salt Lake City, Utah 84112

Gordy, Walter, 1964 (3), Department of Physics, Duke University, Durham, North Carolina 27706

Goudsmit, Samuel Abraham, 1947 (3), Department of Physics, Brookhaven National Laboratory, Upton, New York 11973

Graham, Clarence Henry, 1946 (12), Department of Psychology, Columbia University, New York, New York 10027

Granick, Sam, 1965 (7), Rockefeller University, New York, New York 10021

Green, David Ezra, 1962 (14), Institute for Enzyme Research, University of Wisconsin, 1710 University Avenue, Madison, Wisconsin 53706

Greenberg, Joseph Harold, 1965 (11), Department of Anthropology, Stanford University, Stanford, California 94305

Greenewalt, Crawford Hallock, 1952 (4), E. I. du Pont de Nemours \& Company, Incorporated, Wilmington, Delaware 19898

Greenstein, Jesse Leonard, 1957 (2), California Institute of Technology, Pasadena, California 91109

Gregory, William King, 1927 (6), P. O. Box 35, Woodstock, New York 12498

Griffin, Donald Redfield, 1960 (8), Rockefeller University, New York, New York 10021

Griggs, David Tressel, 1952 (13), Institute of Geophysics and Planetary Physics, University of California, Los Angeles, California 90024

Grobstein, Clifford, 1966 (8), Department of Biology, University of California, San Diego, P. O. Box 109, La Jolla, California 92037

Guilford, Joy Paul, 1954 (12), P. O. Box 1288, Beverly Hills, California 90213

Gunsalus, Irwin Clyde, 1965 (14), Department of Chemistry and Chemical Engineering, University of Illinois, Urbana, Illinois 61801

Gutowsky, Herbert Sander, 1960 (5), Department of Chemistry and Chemical Engineering, University of Illinois, Urbana, Illinois 61801

Hallowell, Alfred Irving, 1961 (11), Department of Anthropology, The University Museum, 33rd and Spruce Streets, Philadelphia, Pennsylvania 19104

Hamburger, Viktor, 1953 (8), Department of Biology, Washington University, St. Louis, Missouri 63130

Hammett, Louis Plack, 1943 (5), Route 4, Box 310, Newton, New Jersey 07860

Hammond, George Simms, 1963 (5), Gates and Crellin Laboratories of Chemistry, California Institute of Technology, Pasadena, California 91109

Handler, Philip, 1964 (14), Department of Biochemistry, Duke University Medical Center, Durham, North Carolina 27706 
Harlow, Harry F., 1951 (12), University of Wisconsin, Regional Primate Research Center, 1223 Capitol Court, Madison, Wisconsin 53706

Harned, Herbert Spencer, 1950 (5), 207 Armory Street, New Haven, Connecticut 06511

Harrar, J. George, 1966 (15), Rockefeller Foundation, 111 West 50th Street, New York, New York 10020

Hartline, Haldan Keffer, 1948 (9), Rockefeller University, New York, New York 10021

Haskins, Caryl Parker, 1956 (8), Carnegie Institution of Washington, $1530 \mathrm{P}$ Street, N. W., Washington, D. C. 20005

Hassid, William Zev, 1958 (14), Department of Biochemistry, Biochemistry Building, University of California, Berkeley, California 94720

Hastings, Albert Baird, 1939 (9), 5912 Bellevue Avenue, La Jolla, California 92037

Haurwitz, Bernhard, 1960 (13), National Center for Atmospheric Research, Boulder, Colorado 80302

Haury, Emil Walter, 1956 (11), Department of Anthropology, University of Arizona, Tucson, Arizona 85721

Hauser, Charles Roy, 1958 (5), Department of Chemistry, Duke University, Durham, North Carolina 27706

Haworth, Leland John, 1965 (3), National Science Foundation, Washington, D. C. 20550

Hedberg, Hollis Dow, 1960 (6), Department of Geology, Princeton University, Princeton, New Jersey 08540

Heidelberger, Michael, 1942 (10), Department of Pathology, New York University School of Medicine, 550 First Avenue, New York, New York 10016

Helliwell, Robert Arthur, 1967 (13), Radioscience Laboratory, Stanford University, Stanford, California 94305

Hendricks, Sterling Brown, 1952 (7), Plant Industry Station, Beltsville, Maryland 20705

Herb, Raymond George, 1955 (3), Department of Physics, University of Wisconsin, Madison, Wisconsin 53706

Herbig, George Howard, 1964 (2), Lick Observatory, University of California, Santa Cruz, California 95060

Herget, Paul, 1962 (2), Cincinnati Observatory, Observatory Place, Cincinnati, Ohio 45208

Herschbach, Dudley Robert, 1967 (5), Department of Chemistry, Harvard University, 12 Oxford Street, Cambridge, Massachusetts 02138

Hershey, Alfred Day, 1958 (7), Genetics Research Unit, Carnegie Institution of Washington, P. O. Box 200, Cold Spring Harbor, New York 11724

Herzfeld, Karl Ferdinand, 1960 (3), Department of Physics, Catholic University of America, Washington, D. C. 20017

Hess, Harry Hammond, 1952 (6), Department of Geology, Princeton University, Princeton, New Jersey 08540

Hewett, Donnel Foster, 1937 (6), 345 Middlefield Road, Menlo Park, California 94025

Hildebrand, Joel Henry, 1929 (5), Department of Chemistry, University of California, Berkeley, California 94720 
Hilgard, Ernest Ropiequet, 1948 (12), Department of Psychology, Stanford University, Stanford, California 94305

Hill, Terrell Leslie, 1965 (5), Division of Natural Sciences, University of California, Santa Cruz, California 95060

Hille, Carl Einar, 1953 (1), 210 Edwards Street, New Haven, Connecticut 06511

Hirschfelder, Joseph Oakland, 1953 (5), Theoretical Chemistry Institute, University of Wisconsin, 1112 West Johnson Street, Madison, Wisconsin 53706

Hirst, George Keble, 1966 (10), Public Health Research Institute of the City of New York, Inc., Foot East 16th Street, New York, New York 10009

Hisaw, Frederick Lee, 1947 (8), The Biological Laboratories, Harvard University, 16 Divinity Avenue, Cambridge, Massachusetts 02138

Hofmann, Klaus Heinrich, 1963 (14), Protein Research Laboratory, University of Pittsburgh School of Medicine, Pittsburgh, Pennsylvania 15213

Hofstadter, Robert, 1958 (3), Department of Physics, Stanford University, Stanford, California 94305

Hollaender, Alexander, 1957 (7), Biology Division, Oak Ridge National Laboratory, P. O. Box Y, Oak Ridge, Tennessee 37830

Holtfreter, Johannes, 1955 (8), Biological Laboratories, University of Rochester, Rochester, New York 14627

Horecker, Bernard Leonard, 1961 (14), Department of Molecular Biology, Albert Einstein College of Medicine, Bronx, New York 10461

Hornig, Donald Frederick, 1957 (5), Executive Office Building, Washington, D. C. 20506

Horsfall, Frank Lappin, Jr., 1948 (10), Sloan-Kettering Institute for Cancer Research, New York, New York 10021

Horsfall, James Gordon, 1953 (7), Connecticut Agricultural Experiment Station, Box 1106, New Haven, Connecticut 06504

Hotchkiss, Rollin Douglas, 1961 (10), Rockefeller University, New York, New York 10021

Hottel, Hoyt Clarke, 1963 (4), Room 12-110, Department of Chemical Engineering, Massachusetts Institute of Technology, Cambridge, Massachusetts 02139

Houston, William Vermillion, 1943 (3), Rice University, Houston, Texas 77001

Howells, William White, 1967 (11), Peabody Museum, Harvard University, Cambridge, Massachusetts 02138

Hubbert, Marion King, 1955 (6), United States Geological Survey, Washington, D. C. 20242

Hubbs, Carl Leavitt, 1952 (8), Scripps Institution of Oceanography, La Jolla, California 92037

Huebner, Robert Joseph, 1960 (10), National Institutes of Health, Bethesda, Maryland 20014

Huggins, Charles Brenton, 1949 (10), The Ben May Laboratory for Cancer Research, University of Chicago, 950 East 59th Street, Chicago, Illinois 60637

Hughes, Vernon Willard, 1967 (3), Department of Physics, Yale University, New Haven, Connecticut 06520

Hunsaker, Jerome Clarke, 1935 (4), Room 33-207, Department of Aeronautics and Astronautics, Massachusetts Institute of Technology, Cambridge, Massachusetts 02139 
Hutchinson, George Evelyn, 1950 (8), Osborn Memorial Laboratories, Yale University, New Haven, Connecticut 06520

Hutchison, Clyde Allen, Jr., 1963 (5), The Enrico Fermi Institute for Nuclear Studies, University of Chicago, Chicago, Illinois 60637

Hyman, Libbie Henrietta, 1961 (8), American Museum of Natural History, Central Park West at 79th Street, New York, New York 10024

Inghram, Mark Gordon, 1961 (3), Department of Physics, University of Chicago, Chicago, Illinois 60637

Ingle, Dwight Joyce, 1963 (9), Department of Physiology, University of Chicago, 951 East 58th Street, Chicago, Illinois 60637

Irwin, Malcolm Robert, 1950 (8), Department of Genetics, University of Wisconsin, Madison, Wisconsin 53706

Iselin, Columbus O'Donnell, 1951 (13), Woods Hole Oceanographic Institution, Woods Hole, Massachusetts 02543

Jacobs, Merkel Henry, 1939 (8), School of Medicine, University of Pennsylvania, Philadelphia, Pennsylvania 19104

Jacobs, Walter Abraham, 1932 (5), 2548 Laurel Pass Road, Los Angeles, California 90046

Jacobson, Leon Orris, 1965 (10), Division of the Biological Sciences, University of Chicago, 950 East 59th Street, Chicago, Illinois 60637

Jacobson, Nathan, 1954 (1), Department of Mathematics, Yale University, New Haven, Connecticut 06520

James, Harold Lloyd, 1962 (6), United States Geological Survey, Washington, D. C. 20242

John, Fritz, 1964 (1), Courant Institute of Mathematical Sciences, New York University, 251 Mercer Street, New York, New York 10012

Johnson, Clarence Leonard, 1965 (4), Lockheed Aircraft Corporation, Burbank, California 91503

Johnson, John Raven, 1948 (5), Deer Valley Farm, Townshend, Vermont 05353

Johnson, William Summer, 1952 (5), Department of Chemistry, Stanford University, Stanford, California 94305

Johnston, Harold Sledge, 1965 (5), Department of Chemistry, University of California, Berkeley, California 94720

Joy, Alfred Harrison, 1944 (2), Mount Wilson and Palomar Observatories, 813 Santa Barbara Street, Pasadena, California 91106

Kabat, Elvin Abraham, 1966 (14), Department of Microbiology, Columbia University College of Physicians and Surgeons, 630 West 168th Street, New York, New York 10032

Kac, Mark, 1965 (1), Rockefeller University, New York, New York 10021

Kalckar, Herman Moritz, 1959 (14), Biochemical Research Department, Massachusetts General Hospital, Boston, Massachusetts 02114

Kamen, Martin David, 1962 (14), Department of Chemistry, Revelle College, University of California, San Diego, La Jolla, California 92037

Kantrowitz, Arthur Robert, 1966 (4), AVCO-Everett Research Laboratory, 2385 Revere Beach Parkway, Everett, Massachusetts 02149

Kaplan, Joseph, 1957 (13), Department of Physics, University of California, Los Angeles, California 90024 
Kaplansky, Irving, 1966 (1), Department of Mathematics, University of Chicago, Chicago, Illinois 60637

Karplus, Martin, 1967 (5), Department of Chemistry, Harvard University, 12 Oxford Street, Cambridge, Massachusetts 02138

Kaufmann, Berwind Petersen, 1952 (7), Department of Zoology, University of Michigan, Ann Arbor, Michigan 48104

Kauzmann, Walter Joseph, 1964 (5), Department of Chemistry, Princeton University, Princeton, New Jersey 08540

Kellogg, Arthur Remington, 1951 (8), 5305 28th Street, N. W., Washington, D. C. 20015

Kelly, Mervin J., 1945 (4), 2 Windemere Terrace, Short Hills, New Jersey 07078

Kemble, Edwin Crawford, 1931 (3), Physics Laboratories, Harvard University, Cambridge, Massachusetts 02138

Kendall, Edward Calvin, 1950 (14), 3 Queenston Place, Princeton, New Jersey 08540

Kennedy, Eugene Patrick, 1964 (14), Department of Biological Chemistry, Harvard Medical School, 25 Shattuck Street, Boston, Massachusetts 02115

Kerst, Donald William, 1951 (3), Physics Department, Sterling Hall, University of Wisconsin, Madison, Wisconsin 53706

Kety, Seymour Solomon, 1962 (9), Laboratory of Clinical Science, National Institute of Mental Health, Bethesda, Maryland 20014

Keyes, Frederick George, 1930 (5), Room 6-123, Massachusetts Institute of Technology, Cambridge, Massachusetts 02139

Khorana, Har Gobind, 1966 (14), Institute for Enzyme Research, University of Wisconsin, 1710 University Avenue, Madison, Wisconsin 53706

Kimball, George Elbert, 1954 (5), Arthur D. Little, Inc., 35 Acorn Park, Cambridge, Massachusetts 02140

King, Charles Glen, 1951 (14), 39 Devon Road, Bronxville, New York 10708

Kinzel, Augustus Braun, 1960 (4), The Salk Institute, P. O. Box 1809, San Diego, California 92112

Kistiakowsky, George Bogdan, 1939 (5), Department of Chemistry, Harvard University, 12 Oxford Street, Cambridge, Massachusetts 02138

Kittel, Charles, 1957 (3), Department of Physics, University of California, Berkeley, California 94720

Klüver, Heinrich, 1957 (12), Culver Hall, University of Chicago, Chicago, Illinois 60637

Knipling, Edward Fred, 1966 (15), Entomology Research Division, Agricultural Research Service, Beltsville, Maryland 20705

Knopoff, Leon, 1963 (13), Institute of Geophysics and Planetary Physics, University of California, Los Angeles, California 90024

Kolthoff, Izaak, Maurits, 1958 (5), School of Chemistry, University of Minnesota, Minneapolis, Minnesota 55455

Kornberg, Arthur, 1957 (14), Department of Biochemistry, Stanford University Medical School, Palo Alto, California 94304

Koshland, Daniel Edward, Jr., 1966 (14), Department of Biochemistry, University of California, Berkeley, California 94720 
Kramer, Paul Jackson, 1962 (7), Department of Botany, Duke University, Durham, North Carolina 27706

Krauskopf, Konrad Bates, 1959 (6), School of Earth Sciences, Stanford University, Stanford, California 94305

Krayer, Otto, 1964 (9), 27 Howland Road, West Newton, Massachusetts 02165

Krogman, Wilton Marion, 1966 (11), Division of Graduate Medicine, School of Medicine, University of Pennsylvania, Philadelphia, Pennsylvania 19104

Kuffler, Stephen William, 1964 (9), Department of Neurobiology, Harvard Medical School, 25 Shattuck Street, Boston, Massachusetts 02115

Kuiper, Gerard Peter, 1950 (2), Lunar and Planetary Laboratory, Space Sciences Building, University of Arizona, Tucson, Arizona 85721

Kunitz, Moses, 1967 (14), Rockefeller University, New York, New York 10021

Kunkel, Henry George, 1967 (10), Rockefeller University, New York, New York 10021

Kusch, Polykarp, 1956 (3), Department of Physics, Columbia University, New York, New York 10027

Lamb, Willis Eugene, Jr., 1954 (3), Sloane Physics Laboratory, Yale University, New Haven, Connecticut 06520

Land, Edwin Herbert, 1953 (3), Polaroid Corporation, Cambridge, Massachusetts 02139

Landis, Eugene Markley, 1954 (9), Department of Physiology, Harvard Medical School, 25 Shattuck Street, Boston, Massachusetts 02115

Lang, Anton, 1967 (7), MSU/AEC Plant Research Laboratory, Michigan State University, East Lansing, Michigan 48823

Lardy, Henry Arnold, 1958 (14), Institute for Enzyme Research, University of Wisconsin, 1702 University Avenue, Madison, Wisconsin 53706

Lauritsen, Charles Christian, 1941 (3), W. K. Kellogg Radiation Laboratory, California Institute of Technology, Pasadena, California 91109

Lederberg, Joshua, 1957 (14), Genetics Department, School of Medicine, Stanford University, Palo Alto, California 94304

Lederman, Leon Max, 1965 (3), Department of Physics, Columbia University, 538 West 120th Street, New York, New York 10027

Lee, Tsung-Dao, 1964 (3), Department of Physics, Columbia University, New York, New York 10027

Lefschetz, Solomon, 1925 (1), Fine Hall, Princeton University, Princeton, New Jersey 08540

Lehninger, Albert Lester, 1956 (14), School of Medicine, Johns Hopkins University, 725 North Wolfe Street, Baltimore, Maryland 21205

Leighton, Robert Benjamin, 1966 (2), Department of Physics, California Institute of Technology, Pasadena, California 91109

Leonard, Nelson Jordan, 1955 (5), Department of Chemistry and Chemical Engineering, University of Illinois, Urbana, Illinois 61801

Leopold, Luna Bergere, 1967 (6), United States Geological Survey, Washington, D.C. 20242

Lerner, I. Michael, 1959 (8), Genetics Department, Mulford Hall, University of California, Berkeley, California 94720 
Levine, Philip, 1966 (8), Immunohematology Division, Ortho Research Foundation, Raritan, New Jersey 08869

Levinson, Norman, 1967 (1), Room 2-365, Department of Mathematics, Massachusetts Institute of Technology, Cambridge, Massachusetts 02139

Lewis, Warren Kendall, 1938 (4), Room 12-112, Massachusetts Institute of Technology, Cambridge, Massachusetts 02139

Lewy, Hans, 1964 (1), Department of Mathematics, 301 Campbell Hall, University of California, Berkeley, California 94720

Libby, Willard Frank, 1950 (5), Department of Chemistry, University of California, 405 Hilgard Avenue, Los Angeles, California 90024

Lim, Robert K. S., (1942) 1965* (9), Medical Sciences Research Laboratory, Miles Laboratories, Inc., Elkhart, Indiana 46514

Lin, Chia-Chiao, 1962 (1), Department of Mathematics, Massachusetts Institute of Technology, Cambridge, Massachusetts 02139

Lindsley, Donald Benjamin, 1952 (12), Department of Psychology, University of California, Los Angeles, California 90024

Link, Karl Paul, 1946 (14), Department of Biochemistry, University of Wisconsin, Madison, Wisconsin 53706

Lipmann, Fritz Albert, 1950 (14), Rockefeller University, New York, New York 10021

Lipscomb, William Nunn, 1961 (5), Department of Chemistry, Harvard University, 12 Oxford Street, Cambridge, Massachusetts 02138

Little, Clarence Cook, 1945 (8), R. F. D. 1, Ellsworth, Maine 04605

Lloyd, David Pierce Caradoc, 1953 (9), Rockefeller University, New York, New York 10021

Loeb, Robert Frederick, 1946 (9), 950 Park Avenue, New York, New York 10028

Long, Cyril Norman Hugh, 1948 (9), Yale University School of Medicine, 333 Cedar Street, New Haven, Connecticut 06510

Long, Esmond Ray, 1946 (10), 220 Locust Street, Apartment 23-B, Philadelphia, Pennsylvania 19106

Long, Franklin A., 1962 (5), 303 Day Hall, Cornell University, Ithaca, New York 14850

Longsworth, Lewis Gibson, 1947 (5), Rockefeller University, New York, New York 10021

Longwell, Chester Ray, 1935 (6), 1820 Mark Twain Street, Palo Alto, California 94303

Loomis, Alfred Lee, 1941 (4), 610 Park Avenue, New York, New York 10021

Loomis, Francis Wheeler, 1949 (3), 804 West Illinois Street, Urbana, Illinois 61801

Lorente de Nó, Rafael, 1950 (9), Rockefeller University, New York, New York 10021

Lovering, Thomas Seward, 1949 (6), Department of Geology, University of Arizona, Tucson, Arizona 85721

Low, Francis Eugene, 1967 (3), Room 6-309, Department of Physics, Massachusetts Institute of Technology, Cambridge, Massachusetts 02139

* Elected a foreign associate in 1942; became a naturalized citizen in 1955 and a member of the Academy in 1965 . 
Lowry, Oliver Howe, 1964 (14), School of Medicine, Washington University, 660 South Euclid, St. Louis, Missouri 63110

Luria, Salvador Edward, 1960 (10), Room 56-423, Department of Biology, Massachusetts Institute of Technology, Cambridge, Massachusetts 02139

Lush, Jay Laurence, 1967 (15), Department of Animal Science, Iowa State University, Ames, Iowa 50010

MacDonald, Gordon James Fraser, 1962 (13), Institute for Defense Analyses, 400 Army Navy Drive, Arlington, Virginia 22202

Mackey, George Whitelaw, 1962 (1), Department of Mathematics, Harvard University, Cambridge, Massachusetts 02133

Mackin, Joseph Hoover, 1963 (6), Department of Geology, University of Texas, Austin, Texas 78712

Mac Lane, Saunders, 1949 (1), Eckhart Hall, University of Chicago, Chicago, Illinois 60637

MacLeod, Colin Munro, 1955 (10), The Commonwealth Fund, 1 East 75th Street, New York, New York 10021

Magoun, Horace Winchell, 1955 (9), Graduate Division, University of California, Los Angeles, California 90024

Mangelsdorf, Paul Christoph, 1945 (15), Botanical Museum, Harvard University, Cambridge, Massachusetts 02138

Mark, Herman Francis, 1961 (5), Polymer Research Institute, Polytechnic Institute of Brooklyn, Brooklyn, New York 11201

Markert, Clement Lawrence, 1967 (8), Department of Biology, Yale University, New Haven, Connecticut 06520

Marshak, Robert Eugene, 1958 (3), Department of Physics and Astronomy, University of Rochester, Rochester, New York 14627

Marvel, Carl Shipp, 1938 (5), Department of Chemistry, University of Arizona, Tucson, Arizona 85721

Matthias, Bernd T., 1965 (3), Department of Physics, University of California, San Diego, La Jolla, California 92038

Mayall, Nicholas Ulrich, 1949 (2), Kitt Peak National Observatory, 950 North Cherry Avenue, P. O. Box 4130, Tucson, Arizona 85717

Mayer, Joseph Edward, 1946 (5), Revelle College, University of California, San Diego, La Jolla, California 92037

Mayer, Maria Goeppert, 1956 (3), Department of Physics, Revelle College, University of California, San Diego, La Jolla, California 92037

Maynard, Leonard Amby, 1944 (14), Savage Hall, Cornell University, Ithaca, New York 14850

Mayr, Ernst, 1954 (8), Museum of Comparative Zoology at Harvard College, Oxford Street, Cambridge, Massachusetts 02138

Mazia, Daniel, 1960 (8), Department of Zoology, University of California, Berkeley, California 94720

McCarty, Maclyn, 1963 (10), Rockefeller University, New York, New York 10021

McClintock, Barbara, 1944 (7), Carnegie Institution of Washington, Cold Spring Harbor, New York 11724

McCollum, Elmer Verner, 1920 (14), 2402 Talbot Road, Baltimore, Maryland 21216 
McConnell, Harden Marsden, 1965 (5), Department of Chemistry, Stanford University, Stanford, California 94305

McDermott, Walsh, 1967 (10), Department of Public Health, Cornell University Medical College, New York, New York 10021

McElroy, William David, 1963 (14), Department of Biology, Johns Hopkins University, Baltimore, Maryland 21218

McElvain, Samuel Marion, 1949 (5), Chemistry Research Building, University of Wisconsin, 1112 West Johnson Street, Madison, Wisconsin 53706

McMaster, Philip Duryeé, 1952 (10), Rockefeller University, New York, New York 10021

McMillan, Edwin Mattison, 1947 (3), Lawrence Radiation Laboratory, University of California, Berkeley, California 94720

McShane, Edward James, 1948 (1), Department of Mathematics, University of Virginia, Charlottesville, Virginia 22901

Mehl, Robert Franklin, 1958 (4), Royal Windsor Apartments, No. 701, 222 Melwood Avenue, Pittsburgh, Pennsylvania 15213

Menzel, Donald Howard, 1948 (2), Harvard College Observatory, 60 Garden Street, Cambridge, Massachusetts 02138

Metcalf, Robert Lee, 1967 (15), Department of Entomology, University of California, Riverside, California 92502

Metz, Charles William, 1948 (8), P. O. Box 714, Woods Hole, Massachusetts 02543

Meyer, Karl, 1967 (14), Belfer Graduate School of Science, Yeshiva University, New York, New York 10033

Meyer, Karl Friedrich, 1940 (10), George Williams Hooper Foundation, University of California Medical Center, San Francisco, California 94122

Michener, Charles Duncan, 1965 (8), Department of Entomology, University of Kansas, Lawrence, Kansas 66045

Miles, Walter Richard, 1933 (12), R. F. D. 3, Box 154, Harvard Terrace, Gales Ferry, Connecticut 06335

Miller, Charles Phillip, 1956 (10), 5757 Kimbark Avenue, Chicago, Illinois 60637

Miller, George Armitage, 1962 (12), Center for Cognitive Studies, Harvard University, 33 Kirkland Street, Cambridge, Massachusetts 02138

Miller, Neal Elgar, 1958 (12), Rockefeller University, New York, New York 10021

Milnor, John Willard, 1963 (1), Fine Hall, Princeton University, P. O. Box 708, Princeton, New Jersey 08540

Minkowski, Rudolph Leo, 1959 (2), Radio Astronomy Laboratory, University of California, Berkeley, California 94720

Mirsky, Alfred Ezra, 1954 (8), Rockefeller University, New York, New York 10021

Montgomery, Deane, 1955 (1), School of Mathematics, The Institute for Advanced Study, Princeton, New Jersey 08540

Moore, John Alexander, 1963 (8), Department of Biological Sciences, 954 Schermerhorn Hall, Columbia University, New York, New York 10027

Moore, Robert Lee, 1931 (1), University of Texas, Austin, Texas 78712

Moore, Stanford, 1960 (14), Rockefeller Institute, New York, New York 10021

Morgan, William Wilson, 1956 (2), Yerkes Observatory, University of Chicago, Williams Bay, Wisconsin 53191 
Morrey, Charles Bradfield, Jr., 1962 (1), Department of Mathematics, University of California, Berkeley, California 94720

Morse, Harold Marston, 1932 (1), The Institute for Advanced Study, Princeton, New Jersey 08540

Morse, Philip McCord, 1955 (3), Room 24-215, Department of Physics, Massachusetts Institute of Technology, Cambridge, Massachusetts 02139

Mountcastle, Vernon Benjamin, 1966 (9), Department of Physiology, School of Medicine, Johns Hopkins University, 725 North Wolfe Street, Baltimore, Maryland 21205

Movius, Hallam Leonard, Jr., 1957 (11), Peabody Museum, Harvard University, Cambridge, Massachusetts 02138

Mulliken, Robert Sanderson, 1936 (3), Laboratory of Molecular Structure and Spectra, Department of Physics, University of Chicago, Chicago, Illinois 60637

Münch, Guido, 1967 (2), Mount Wilson and Palomar Observatories, 1201 East California Boulevard, Pasadena, California 91109

Munk, Walter Heinrich, 1956 (13), Institute of Geophysics and Planetary Physics, University of California, San Diego, La Jolla, California 92037

Murdock, George Peter, 1964 (11), Department of Anthropology, University of Pittsburgh, Pittsburgh, Pennsylvania 15213

Murnaghan, Francis Dominic, 1942 (1), 6202 Sycamore Road, Baltimore, Maryland 21212

Nachmansohn, David, 1965 (14), Columbia University College of Physicians and Surgeons, 630 West 168th Street, New York, New York 10032

Nauta, Walle Jetze Harinx, 1967 (12), Room E10-104, Department of Psychology, Massachusetts Institute of Technology, Cambridge, Massachusetts 02139

Neel, James Van Gundia, 1963 (8), Department of Human Genetics, University of Michigan Medical School, Ann Arbor, Michigan 48104

Neff, William Duwayne, 1964 (12), Center for Neural Sciences, Indiana University, Bloomington, Indiana 47401

Neurath, Hans, 1961 (14), Department of Biochemistry, University of Washington, Seattle, Washington 98105

Newman, Melvin Spencer, 1956 (5), Ohio State University, Columbus, Ohio 43210

Newmark, Nathan Mortimore, 1966 (4), Department of Civil Engineering, 1114 Civil Engineering Building, University of Illinois, Urbana, Illinois 61801

Neyman, Jerzy, 1963 (1), Department of Statistics, University of California, Berkeley, California 94720

Nier, Alfred Otto C., 1950 (3), School of Physics and Astronomy, University of Minnesota, Minneapolis, Minnesota 55455

Nirenberg, Marshall Warren, 1967 (14), Laboratory of Biochemical Genetics, National Heart Institute, Bethesda, Maryland 20014

Nolan, Thomas Brennan, 1951 (6), 2219 California Street, N. W., Washington, D. C. 20008

Northrop, John Howard, 1934 (14), 3510 Life Sciences Building, University of California, Berkeley, California 94720

Noyes, William Albert, Jr., 1943 (5), Department of Chemistry, University of Texas, Austin, Texas 78712 
O'Brien, Brian, 1954 (3), Box 52, North Woodstock, Connecticut 06257

Ochoa, Severo, 1957 (14), New York University School of Medicine, 550 First Avenue, New York, New York 10016

Olds, James, 1967 (12), Department of Psychology, University of Michigan, Ann Arbor, Michigan 48104

Olson, Harry Ferdinand, 1959 (4), RCA Laboratories, David Sarnoff Research Center, Princeton, New Jersey 08541

Oncley, John Lawrence, 1947 (14), Biophysics Research Division, Institute of Science and Technology, University of Michigan, Ann Arbor, Michigan 48104

Onsager, Lars, 1947 (5), Sterling Chemistry Laboratory, Yale University, New Haven, Connecticut 06520

Opie, Eugene Lindsay, 1923 (10), Rockefeller University, New York, New York 10021

Osterbrock, Donald Edward, 1966 (2), Washburn Observatory, University of Wisconsin, Madison, Wisconsin 53706

Owen, Ray David, 1966 (8), Division of Biology, California Institute of Technology, Pasadena, California 91109

Painter, Theophilus Shickel, 1938 (8), University of Texas, Austin, Texas 78712

Pais, Abraham, 1962 (3), Rockefeller University, New York, New York 10021

Palade, George Emil, 1961 (8), Rockefeller University, New York, New York 10021

Panofsky, Wolfgang K. H., 1954 (3), Stanford Linear Accelerator Center, Stanford University, P. O. Box 4349, Stanford, California 94305

Pappenheimer, John Richard, 1965 (9), Department of Physiology, Harvard Medical School, 25 Shattuck Street, Boston, Massachusetts 02115

Parker, Eugene Newman, 1967 (2), Laboratory for Astrophysics, 933 East 56th Street, Chicago, Illinois 60637

Patterson, Bryan, 1963 (6), Museum of Comparative Zoology at Harvard College, Oxford Street, Cambridge, Massachusetts 02138

Paul, John Rodman, 1945 (10), Yale University School of Medicine, 333 Cedar Street, New Haven, Connecticut 06510

Pauling, Linus, 1933 (5), Big Sur, California 93920

Pecora, William Thomas, 1965 (6), United States Geological Survey, Washington, D. C. 20242

Pekeris, Chaim Leib, 1952 (13), Department of Applied Mathematics, The Weizmann Institute of Science, Rehovot, Israel

Perlman, Isadore, 1963 (5), Lawrence Radiation Laboratory, Berkeley, California 94720

Pettijohn, Francis John, 1966 (6), Department of Geology, Johns Hopkins University, Baltimore, Maryland 21218

Pfaffmann, Carl, 1959 (12), Rockefeller University, New York, New York 10021

Pickering, William Hayward, 1962 (4), Jet Propulsion Laboratory, 4800 Oak Grove Drive, Pasadena, California 91103

Pierce, John Robinson, 1955 (4), Bell Telephone Laboratories, Incorporated, Murray Hill, New Jersey 07971

Piggot, Charles Snowden, 1946 (13), Holly Grove, Route 2, Box 68, Lexington Park, Maryland 20653 
Pimentel, George Claude, 1966 (5), Department of Chemistry, University of California, Berkeley, California 94720

Pincus, Gregory Goodwin, 1965 (9), Worcester Foundation for Experimental Biology, Shrewsbury, Massachusetts 01545

Piore, Emanuel Ruben, 1963 (4), International Business Machines Corporation, Armonk, New York 10504

Pittendrigh, Colin Stephenson, 1963 (8), Princeton University Graduate School, 1'. O. Box 255, Princeton, New Jersey 08540

Pitts, Robert Franklin, 1956 (9), Cornell University Medical College, 1300 York Avenue, New York, New York 10021

Pitzer, Kenneth Sanborn, 1949 (5), Rice University, Houston, Texas 77001

Porter, Keith Roberts, 1964 (8), The Biological Laboratories, Harvard University, 16 Divinity Avenue, Cambridge, Massachusetts 02138

Pound, Robert Vivian, 1961 (3), Lyman Laboratory of Physics, Harvard University, Cambridge, Massachusetts 02138

Press, Frank, 1958 (13), Department of Geology and Geophysics, Massachusetts Institute of Technology, Cambridge, Massachusetts 02139

Puck, Theodore Thomas, 1960 (10), Department of Biophysics, University of Colorado Medical Center, Denver, Colorado 80220

Purcell, Edward Mills, 1951 (3), Lyman Laboratory of Physics, Harvard University, Cambridge, Massachusetts 02138

Rabi, Isidor Isaac, 1940 (3), Columbia University, New York, New York 10027

Racker, Efraim, 1966 (14), Section of Biochemistry and Molecular Biology, Cornell University, Ithaca, New York 14850

Ramsey, Norman Foster, 1952 (3), Lyman Laboratory of Physics, Harvard University, Cambridge, Massachusetts 02138

Raper, John Robert, 1964 (7), The Biological Laboratories, Harvard University, 16 Divinity Avenue, Cambridge, Massachusetts 02138

Raper, Kenneth Bryan, 1949 (7), Department of Bacteriology, University of Wisconsin, Madison, Wisconsin 53706

Ratliff, Floyd, 1966 (12), Rockefeller University, New York, New York 10021

Raymond, Arthur Emmons, 1950 (4), 73 Oakmont Drive, Los Angeles, California 90049

Redfield, Alfred Clarence, 1958 (13), P. O. Box 106, Woods Hole, Massachusetts 02543

Reichelderfer, Francis Wilton, 1945 (13), 3031 Sedgwick Street, N. W., Washington, D. C. 20008

Revelle, Roger Randall, 1957 (13), Harvard Center for Population Studies, 9 Bow Street, Cambridge, Massachusetts 02138

Rhoades, Marcus Morton, 1946 (7), Department of Botany, Indiana University, Bloomington, Indiana 47401

Rice, Oscar Knefler, 1964 (5), Department of Chemistry, University of North Carolina, Chapel Hill, North Carolina 27514

Rich, Arnold Rice, 1954 (10), 14 Edgevale Road, Baltimore, Maryland 21210

Richards, Dickinson W., 1958 (9), Columbia University College of Physicians and Surgeons, 630 West 168th Street, New York, New York 10032 
Richter, Curt Paul, 1948 (12), Johns Hopkins Hospital, Baltimore, Maryland 21205

Rick, Charles Madeira, Jr., 1967 (15), Department of Vegetable Crops, University of California, Davis, California 95616

Riddle, Oscar, 1939 (8), Route 4, Box 576, Plant City, Florida 33566

Riggs, Lorrin Andrews, 1961 (12), Hunter Laboratory of Psychology, Brown University, Providence, Rhode Island 02912

Riker, Albert Joyce, 1951 (7), 3810 Manitou Way, Madison, Wisconsin 53711

Rittenberg, David, 1953 (14), Columbia University College of Physicians and Surgeons, 630 West 168th Street, New York, New York 10032

Robbins, William Jacob, 1940 (7), 301 East 66th Street, New York, New York 10021

Roberts, John D., 1956 (5), Gates and Crellin Laboratories of Chemistry, California Institute of Technology, Pasadena, California 91109

Roberts, Richard Brooke, 1961 (7), Department of Terrestrial Magnetism, Carnegie Institution of Washington, 5241 Broad Branch Road, N. W., Washington, D. C. 20015

Roeder, Kenneth David, 1964 (8), Department of Biology, Tufts University, Medford, Massachusetts 02155

Romer, Alfred Sherwood, 1944 (8), Museum of Comparative Zoology, Harvard University, Oxford Street, Cambridge, Massachusetts 02138

Rose, William Cumming, 1936 (14), 710 West Florida Avenue, Urbana, Illinois 61801

Rossi, Bruno Benedetto, 1950 (3), Room 26-569, Department of Physics, Massachusetts Institute of Technology, Cambridge, Massachusetts 02139

Rossini, Frederick Dominic, 1951 (5), University of Notre Dame, Notre Dame. Indiana 46556

Rous, Francis Peyton, 1927 (10), Rockefeller University, New York, New York 10021

Rouse, Irving, 1962 (11), Department of Anthropology, Yale University, Box 2114. Yale Station, New Haven, Connecticut 06520

Rubey, William Walden, 1945 (6), Department of Geology and Institute of Geophysics, University of California, Los Angeles, California 90024

Russell, Richard Joel, 1959 (6), Coastal Studies Institute, Louisiana State University, Baton Rouge, Louisiana 70803

Sabin, Albert Bruce, 1951 (10), The Children's Hospital Research Foundation, University of Cincinnati College of Medicine, Elland Avenue and Bethesda, Cincinnati, Ohio 45229

Salpeter, Edwin Ernest, 1967 (2), Laboratory of Nuclear Studies, Cornell University, Ithaca, New York 14850

Sandage, Allan Rex, 1963 (2), Mount Wilson and Palomar Observatories, 813 Santa Barbara Street, Pasadena, California 91106

Savage, John Lucian, 1949 (4), Park Manor, 1801 East 19th Street, Denver, Colorado 80218

Sax, Karl, 1941 (7), Bishop Hollow Road, Media, Pennsylvania 19063

Scatchard, George, 1946 (5), Department of Chemistry, Massachusetts Institute of Technology, Cambridge, Massachusetts 02139 
Schairer, John Frank, 1953 (6), Geophysical Laboratory, Carnegie Institution of Washington, 2801 Upton Street, N. W., Washington, D. C. 20008

Scharrer, Berta Vogel, 1967 (8), Department of Anatomy, Albert Einstein College of Medicine, Bronx, New York 10461

Scheraga, Harold Abraham, 1966 (5), Department of Chemistry, Cornell University, Ithaca, New York 14850

Schiff, Leonard Isaac, 1957 (3), Department of Physics, Stanford University, Stanford, California 94305

Schmidt, Carl Frederic, 1949 (9), Aviation Medical Research Department, United States Naval Air Development Center, Johnsville, Warminster, Pennsylvania 18974

Schmidt-Nielsen, Knut, 1963 (8), Department of Zoology, Duke University, Durham, North Carolina 27706

Schmitt, Francis Otto, 1948 (8), Room 16-512, Department of Biology, Massachusetts Institute of Technology, Cambridge, Massachusetts 02139

Scholander, Per Fredrik, 1961 (8), Scripps Institution of Oceanography, University of California, La Jolla, California 92037

Schwarzschild, Martin, 1956 (2), Princeton University Observatory, Peyton Hall, Princeton, New Jersey 08541

Schwinger, Julian, 1949 (3), Department of Physics, Harvard University, Cambridge, Massachusetts 02138

Seaborg, Glenn Theodore, 1948 (5), United States Atomic Energy Commission, Washington, D. C. 20545

Sears, Ernest Robert, 1964 (15), United States Department of Agriculture, 108 Curtis Hall, University of Missouri, Columbia, Missouri 65201

Segrè, Emilio, 1952 (3), Department of Physics, University of California, Berkeley, California 94720

Seitz, Frederick, 1951 (3), University of Illinois, Urbana, Illinois 61801; National Academy of Sciences, Washington, D. C. 20418

Serber, Robert, 1952 (3), Department of Physics, Columbia University, New York, New York 10027

Shane, Charles Donald, 1961 (2), P. O. Box 582, Santa Cruz, California 95061

Shannon, Claude Elwood, 1956 (1), Research Laboratory of Electronics, Massachusetts Institute of Technology, Cambridge, Massachusetts 02139

Shannon, James Augustine, 1965 (10), National Institutes of Health, Bethesda, Maryland 20014

Shapiro, Ascher Herman, 1967 (4), Room 3-174, Department of Mechanical Engineering, Massachusetts Institute of Technology, Cambridge, Massachusetts 02139

Shapiro, Harry Lionel, 1949 (11), American Museum of Natural History, Central Park West at 79th Street, New York, New York 10024

Shapley, Harlow, 1924 (2), Sharon Cross Road, Peterboro, New Hampshire 03458

Shedlovsky, Theodore, 1953 (5), Rockefeller University, New York, New York 10021

Sheehan, John Clark, 1957 (5), Department of Chemistry, Massachusetts Institute of Technology, Cambridge, Massachusetts 02139 
Shemin, David, 1958 (14), Columbia University College of Physicians and Surgeons, 630 West 168th Street, New York, New York 10032

Sherwood, Thomas Kilgore, 1958 (4), Room 12-186, Department of Chemical Engineering, Massachusetts Institute of Technology, Cambridge, Massachusetts 02139

Shockley, William, 1951 (4), Stanford Electronics Laboratory, Stanford University, Stanford, California 94305

Simon, Herbert Alexander, 1967 (12), Graduate School of Industrial Administration, Carnegie Institute of Technology, Pittsburgh, Pennsylvania 15213

Simpson, George Gaylord, 1941 (6), Department of Geology, University of Arizona, Tucson, Arizona 85721

Simpson, John Alexander, 1959 (3), The Enrico Fermi Institute for Nuclear Studies, University of Chicago, Chicago, Illinois 60637

Sinnott, Edmund Ware, 1936 (7), 459 Prospect Street, New Haven, Connecticut 06511

Sinsheimer, Robert Louis, 1967 (14), Division of Biology, California Institute of Technology, Pasadena, California 91109

Skinner, Burrhus Frederic, 1950 (12), William James Hall, Harvard University, 33 Kirkland Street, Cambridge, Massachusetts 02138

Skoog, Folke Karl, 1956 (7), Department of Botany, Birge Hall, University of Wisconsin, Madison, Wisconsin 53706

Slater, John Clarke, 1932 (3), 522 Nuclear Sciences Building, University of Florida, Gainesville, Florida 32601

Slepian, Joseph, 1941 (4), 1115 Lancaster Street, Pittsburgh, Pennsylvania 15218

Slichter, Charles Pence, 1967 (3), Department of Physics, University of Illinois, Urbana, Illinois 61801

Slichter, Louis Byrne, 1944 (13), Institute of Geophysics, University of California, Los Angeles, California 90024

Slipher, Vesto Melvin, 1921 (2), P. O. Box 326, Flagstaff, Arizona 86001

Smith, Albert Charles, 1963 (7), University of Hawaii, Honolulu, Hawaii 96822

Smith, Cyril Stanley, 1957 (4), Room 14N-317, Massachusetts Institute of Technology, Cambridge, Massachusetts 02139

Smith, Emil L., 1962 (14), Department of Biological Chemistry, University of California School of Medicine, Los Angeles, California 90024

Smith, Lee Irvin, 1944 (5), 2323 West 52nd Street, Minneapolis, Minnesota 55410

Smith, Paul Althaus, 1947 (1), Department of Mathematics, Columbia University, New York, New York 10027

Smith, Philip Edward, 1939 (8), 2449 Pine Knoll Drive, Walnut Creek, California 94529

Smyth, Charles Phelps, 1955 (5), Frick Chemical Laboratory, Princeton University, Princeton, New Jersey 08540

Snell, Esmond Emerson, 1955 (14), Department of Biochemistry, University of California, Berkeley, California 94720

Soderberg, Carl Richard, 1947 (4), Room 1-207, Massachusetts Institute of Technology, Cambridge, Massachusetts 02139

Sonneborn, Tracy Morton, 1946 (8), 220 Jordan Hall, Department of Zoology, Indiana University, Bloomington, Indiana 47401 
Spedding, Frank Harold, 1952 (5), Ames Laboratory of the AEC, Iowa State University, Ames, Iowa 50010

Spencer, Donald Clayton, 1961 (1), Department of Mathematics, Stanford University, Stanford, California 94305

Sperry, Roger Wolcott, 1960 (12), Division of Biology, California Institute of Technology, Pasadena, California 91109

Spiegelman, Sol, 1965 (14), Department of Microbiology, University of Illinois, Urbana, Illinois 61801

Spitzer, Lyman, Jr., 1952 (2), Princeton University Observatory, Peyton Hall, Princeton, New Jersey 08540

Sporn, Philip, 1962 (4), American Electric Power Company, Inc., 2 Broadway, New York, New York 10004

Stakman, Elvin Charles, 1934 (7), Institute of Agriculture, University of Minnesota, St. Paul, Minnesota 55101

Stanley, Wendell Meredith, 1941 (14), Virus Laboratory, University of California, Berkeley, California 94720

Stebbins, George Ledyard, 1952 (7), Department of Genetics, University of California, Davis, California 95616

Steenrod, Norman Earl, 1956 (1), Fine Hall, Princeton University, P. O. Box 708, Princeton, New Jersey 08540

Stein, William Howard, 1960 (14), Rockefeller University, New York, New York 10021

Steinberger, Jack, 1966 (3), Department of Physics, Columbia University, New York, New York 10027

Stephens, Stanley George, 1967 (15), Department of Genetics, North Carolina State University, Box 5487, Raleigh, North Carolina 27607

Stern, Curt, 1948 (8), Department of 'Zoology, University of California, Berkeley. California 94720

Stern, Otto, 1945 (3), 759 Cragmont Avenue, Berkeley, California 94708

Stevens, Stanley Smith, 1946 (12), Laboratory of Psychophysics, Harvard University, 33 Kirkland Street, Cambridge, Massachusetts 02138

Steward, Julian H., 1954 (11), Department of Anthropology, 109 Davenport Hall, University of illinois, Urbana, Illinois 61801

Stewart, Thomas Dale, 1962 (11), Museum of Natural History, Smithsonian Institution, Washington, D. C. 20560

Stockmayer, Walter Hugo, 1956 (5), Department of Chemistry, Dartmouth C'ollege, Hanover, New Hampshire 03755

Stoker, James Johnston, 1963 (1), Courant Institute of Mathematical Sciences, New York University, 251 Mercer Street, New York, New York 10012

Stommel, Henry Melson, 1961 (13), Room 54-1416, Massachusetts Institute of Technology, Cambridge, Massachusetts 02139

Stone, Marshall Harvey, 1938 (1), 303 Eckhart Hall, University of Chicago, Chicago, Illinois 60637

Stone, Wilson Stuart, 1960 (8), Genetics Foundation, University of Texas, Austin, Texas 78712

Stork, Gilbert, 1960 (5), Department of Chemistry, Columbia University, New York, New York 10027 
Stratton, Julius Adams, 1950 (4), Ford Foundation, 477 Madison Avenue, New York, New York 10022

Straus, William Louis, Jr., 1962 (8), Department of Anatomy, School of Mcdicine, Johns Hopkins University, 725 North Wolfe Street, Baltimore, Maryland 21205

Street, Jabez Curry, 1953 (3), Lyman Laboratory of Physics, Harvard University, Cambridge, Massachusetts 02138

Sturtevant, Alfred Henry, 1930 (8), Division of Biology, California Institute of Technology, Pasadena, California 91109

Suess, Hans Eduard, 1966 (13), Department of Chemistry, University of California, San Diego, La Jolla, California 92037

Suits, Chauncey Guy, 1946 (4), Crosswinds, Pilot Knob, New York, 12844

Sutherland, Earl Wilbur, Jr., 1966 (14), Department of Physiology, School of Medicine, Vanderbilt University, Nashville, Tennessee 37203

Szent-Györgyi, Albert, 1956 (14), Institute for Muscle Research, P. O. Box 187, Woods Hole, Massachusetts 02543

Taliaferro, William Hay, 1940 (10), Argonne National Laboratory, 9700 South Cass Avenue, Argonne, Illinois 60439

Tarbell, Dean Stanley, 1959 (5), Department of Chemistry, Vanderbilt University, Nashville, Tennessee 37203

Tarski, Alfred, 1965 (1), Department of Mathematics, University of California, Berkeley, California 94720

Tatum, Edward Lawrie, 1952 (14), Rockefeller University, New York, New York 10021

Taube, Henry, 1959 (5), Department of Chemistry, Stanford University, Stanford, California 94305

Teller, Edward, 1948 (3), Lawrence Radiation Laboratory, University of California, Berkeley, California 94720

Terman, Frederick Emmons, 1946 (4), 174 McCullough Building, Stanford University, Stanford, California 94305

Thimann, Kenneth Vivian, 1948 (7), Crown College, University of California, Santa Cruz, California 95060

Thomas, Charles Allen, 1948 (4), Monsanto Company, 800 North Lindbergh Boulevard, St. Louis, Missouri 63166

Thomas, Llewcllyn Hilleth, 1958 (3), Watson Scientific Laboratory, 612 West 115th Street, New York, New York 10025

Thomas, Tracy Yerkes, 1941 (1), Department of Engineering, University of California, Los Angeles, California 90024

Thompson, James Burleigh, Jr., 1967 (6), Department of Geological Sciences, Harvard University, Cambridge, Massachusetts 02138

Tillett, William Smith, 1951 (10), New York University School of Medicine, 550 First Avenue, New York, New York 10016

Timoshenko, Stephen Prokop, 1940 (4), 56 Wuppertal-Elberfeld, Böcklinstrasse 35, Germany

Tishler, Max, 1953 (5), Merck \& Co., Inc., Rahway, New Jersey 07065

Tousey, Richard, 1960 (2), United States Naval Research Laboratory (Code 7140), Washington, D. C. 20390 
Townes, Charles Hard, 1956 (3), Room 6-207, Massachusetts Institute of Technology, Cambridge, Massachusetts 02139

Tukey, John Wilder, 1961 (1), Fine Hall, Princeton University, P. O. Box 708, Princeton, New Jersey 08540

Turkevich, Anthony Leonid, 1967 (5), The Enrico Fermi Institute for Nuclear Studies, University of Chicago, Chicago, Illinois 60637

Turner, Francis John, 1956 (6), Department of Geology and Geophysics, University of California, Berkeley, California 94720

Turner, Richard Baldwin, 1964 (5), Department of Chemistry, Rice University, Houston, Texas 77001

Tuve, Merle Antony, 1946 (3), Carnegie Institution of Washington, 5241 Broad Branch Road, N. W., Washington, D. C. 20015

Uhlenbeck, George Eugene, 1955 (3), Rockefeller University, New York, New York 10021

Ulam, Stanislaw Marcin, 1966 (1), Department of Mathematics, University of Colorado, Boulder, Colorado 80304

Urey, Harold Clayton, 1935 (5), University of California, San Diego, La Jolla, California 92037

Van Allen, James Alfred, 1959 (13), Department of Physics and Astronomy, University of Iowa, Iowa City, Iowa 52240

Van Niel, Cornelis Bernardus, 1945 (7), Hopkins Marine Station of Stanford University, Pacific Grove, California 93950

Van Slyke, Donald Dexter, 1921 (14), Brookhaven National Laboratory, Upton, New York 11973

Van Vleck, John Hasbrouck, 1935 (3), Lyman Laboratory of Physics, Harvard University, Cambridge, Massachusetts 02138

Verhoogen, John, 1956 (13), Department of Geology and Geophysics, University of California, Berkeley, California 94720

Vestine, Ernest Harry, 1954 (13), Rand Corporation, 1700 Main Street, Santa Monica, California 90406

Vickery, Hubert Bradford, 1943 (14), Connecticut Agricultural Experiment Station, New Haven, Connecticut 06504

Villard, Oswald Garrison, Jr., 1958 (13), Radioscience Laboratory, Stanford University, Stanford, California 94305

Visscher, Maurice Bolks, 1956 (9), Department of Physiology, University of Minnesota, Minneapolis, Minnesota 55455

von Békésy, Georg, 1956 (12), Laboratory of Sensory Sciences, University of Hawaii, 1993 East-West Road, Honolulu, Hawaii 96822

Waksman, Selman Abraham, 1942 (10), Institute of Microbiology, Rutgers, The State University, New Brunswick, New Jersey 08903

Wald, George, 1950 (14), The Biological Laboratories, Harvard University, 16 Divinity Avenue, Cambridge, Massachusetts 02138

Walker, John Charles, 1945 (7), Russell Laboratories, University of Wisconsin, Madison, Wisconsin 53706

Wall, Frederick Theodore, 1961 (5), Department of Chemistry, University of California, San Diego, La Jolla, California 92037 
Walling, Cheves Thomson, 1964 (5), Department of Chemistry, Columbia University, New York, New York 10027

Walsh, Joseph Leonard, 1936 (1), Department of Mathematics, University of Maryland, College Park, Maryland 20740

Wangensteen, Owen H., 1966 (10), Department of Surgery, 511 Bio-Medical Library, Diehl Hall, University Medical Center, Minneapolis, Minnesota 55455

Warner, John Christian, 1956 (5), Neville House, Apt. 64, 552 North Neville Street, Pittsburgh, Pennsylvania 15213

Warren, Shields, 1962 (10), Cancer Research Institute, New England Deaconess Hospital, 194 Pilgrim Road, Boston, Massachusetts 02215

Washburn, Sherwood Larned, 1963 (11), Department of Anthropology, University of California, Berkeley, California 94720

Waters, Aaron Clement, 1964 (6), Crown College, University of California, Santa Cruz, California 95060

Watson, Cecil James, 1959 (10), University of Minnesota Medical Unit, Northwestern Hospital, Minneapolis, Minnesota 55407

Watson, James Dewey, 1962 (14), The Biological Laboratories, Harvard University, 16 Divinity Avenue, Cambridge, Massachusetts 02138

Weber, Ernst, 1965 (4), Polytechnic Institute of Brooklyn, 333 Jay Street, Brooklyn, New York 11201

Webster, David Locke, 1923 (3), 1830 Cowper Street, Palo Alto, California 94301

Wedel, Waldo Rudolph, 1965 (11), Office of Anthropology, Museum of Natural History, Smithsonian Institution, Washington, D. C. 20560

Weinberg, Alvin Martin, 1961 (3), Oak Ridge National Laboratory, P. O. Box X, Oak Ridge, Tennessee 37831

Weiss, Paul Alfred, 1947 (8), Rockefeller University, New York, New York 10021

Weisskopf, Victor Frederick, 1952 (3), Department of Physics, Massachusetts Institute of Technology, Cambridge, Massachusetts 02139

Weissman, Samuel Isaac, 1966 (5), Department of Chemistry, Louderman Hall, Washington University, St. Louis, Missouri 63130

Weller, Thomas Huckle, 1964 (10), Department of Tropical Public Health, Harvard School of Public Health, 25 Shattuck Street, Boston, Massachusetts 02115

Went, Frits Warmolt, 1947 (7), Desert Research Institute, University of Nevada, Reno, Nevada 89507

Wentzel, Gregor, 1959 (3), The Enrico Fermi Institute for Nuclear Studies, University of Chicago, Chicago, Illinois 60637

Westheimer, Frank Henry, 1954 (5), Department of Chemistry, Harvard University, 12 Oxford Street, Cambridge, Massachusetts 02138

Wetmore, Alexander, 1945 (8), Smithsonian Institution, Washington, D. C. 20560

Wetmore, Ralph Hartley, 1954 (7), The Biological Laboratories, Harvard University, 16 Divinity Avenue, Cambridge, Massachusetts 02138

Wever, Ernest Glen, 1940 (12), 1S14 Green Hall, Princeton University, Princeton, New Jersey 08540

Wheeler, John Archibald, 1952 (3), Palmer Physical Laboratory, Princeton University, Princeton, New Jersey 08540 
Whipple, Fred Lawrence, 1959 (2), Astrophysical Observatory, Smithsonian Institution, 60 Garden Street, Cambridge, Massachusetts 02138

Whipple, George Hoyt, 1929 (10), School of Medicine and Dentistry, University of Rochester, 260 Crittenden Boulevard, Rochester, New York 14620

Whitford, Albert Edward, 1954 (2), Lick Observatory, University of California, Santa Cruz, California 95060

Whitney, Hassler, 1945 (1), The Institute for Advanced Study, Princeton, New Jersey 08540

Whyburn, Gordon Thomas, 1951 (1), Department of Mathematics, University of Virginia, Charlottesville, Virginia 22903

Wiberg, Kenneth Berle, 1967 (5), Department of Chemistry, Yale University, New Haven, Connecticut 06520

Wick, Gian-Carlo, 1963 (3), Department of Physics, Columbia University, New York, New York 10027

Wiesner, Jerome Bert, 1960 (4), Massachusetts Institute of Technology, Cambridge, Massachusetts 02139

Wigner, Eugene Paul, 1945 (3), Palmer Physical Laboratory, Princeton University, Princeton, New Jersey 08540

Wilder, Raymond Louis, 1963 (1), Department of Mathematics, University of Michigan, Ann Arbor, Michigan 48104

Willey, Gordon Randolph, 1960 (11), Peabody Museum, Harvard University, Cambridge, Massachusetts 02138

Williams, Carroll Milton, 1960 (8), The Biological Laboratories, Harvard University, 16 Divinity Avenue, Cambridge, Massachusetts 02138

Williams, Howel, 1950 (6), Department of Geology and Geophysics, University of California, Berkeley, California 94720

Williams, John Warren, 1952 (5), Chemistry Research Building, University of Wisconsin, 1112 West Johnson Street, Madison, Wisconsin 53706

Williams, Robley Cook, 1955 (14), Department of Molecular Biology, University of California, Berkeley, California 94720

Williams, Roger John, 1946 (14), Clayton Foundation Biochemical Institute, University of Texas, Austin, Texas 78712

Willier, Benjamin Harrison, 1945 (8), Department of Biology, Johns Hopkins University, Baltimore, Maryland 21218

Wilson, Edgar Bright, Jr., 1947 (5), Department of Chemistry, Harvard University, 12 Oxford Street, Cambridge, Massachusetts 02138

Wilson, Olin Chaddock, 1960 (2), Mount Wilson and Palomar Observatories, 813 Santa Barbara Street, Pasadena, California 91106

Wilson, Perry William, 1955 (7), Department of Bacteriology, University of Wisconsin, Madison, Wisconsin 53706

Wilson, Robert Rathbun, 1957 (3), Laboratory of Nuclear Studies, Cornell University, Ithaca, New York 14850

Winstein, Saul, 1955 (5), Department of Chemistry, University of California, Los Angeles, California 90024

Wintersteiner, Oskar, 1950 (14), Squibb Institute for Medical Research, New Brunswick, New Jersey 08903 
Wolfrom, Melville Lawrence, 1950 (14), Department of Chemistry, The Ohio State University, 88 West 18th Avenue, Columbus, Ohio 43210

Wolman, Abel, 1963 (4), 513 Ames Hall, Johns Hopkins University, Baltimore, Maryland 21218

Wood, Harland Goff, 1953 (14), Department of Biochemistry, School of Medicine, Western Reserve University, Cleveland, Ohio 44106

Wood, William Barry, Jr., 1959 (10), School of Medicine, Johns Hopkins University, 725 North Wolfe Street, Baltimore, Maryland 21205

Woodring, Wendell Phillips, 1946 (6), United States National Museum, Washington, D. C. 20560

Woodward, Robert Burns, 1953 (5), Department of Chemistry, Harvard University, 12 Oxford Street, Cambridge, Massachusetts 02138

Woolsey, Clinton Nathan, 1960 (9), Laboratory of Neurophysiology, 283 Medical Sciences Building, University of Wisconsin, Madison, Wisconsin 53706

Wright, Sewall Green, 1934 (8), Department of Genetics, University of Wisconsin, Madison, Wisconsin 53706

Wu, Chien-Shiung, 1958 (3), Department of Physics, Columbia University, New York, New York 10027

Wulf, Oliver Reynolds, 1949 (13), Gates and Crellin Laboratories of Chemistry, California Institute of Technology, Pasadena, California 91109

Wyckoff, Ralph Walter Graystone, 1949 (5), Department of Physics, University of Arizona, Tucson, Arizona 85721

Yang, Chen Ning, 1965 (3), Department of Physics, State University of New York, Stony Brook, New York 11790

Yanofsky, Charles, 1966 (14), Department of Biological Sciences, Stanford University, Stanford, California 94305

Yoder, Hatten Schuyler, Jr., 1958 (6), Geophysical Laboratory, Carnegie Institution of Washington, 2801 Upton Street, N. W., Washington, D. C. 20008

Yost, Don Merlin Lee, 1944 (5), California Institute of Technology, Pasadena. California 91109

Young, William Gould, 1951 (5), Office of the Chancellor, University of California, Los Angeles, California 90024

Zacharias, Jerrold Reinach, 1957 (3), Department of Physics, Massachusetts Institute of Technology, Cambridge, Massachusetts 02139

Zachariasen, Frederik William Houlder, 1949 (3), Department of Physics, University of Chicago, Chicago, Illinois 60637

Zariski, Oscar, 1944 (1), Department of Mathematics, Harvard University, 2 Divinity Avenue, Cambridge, Massachusetts 02138

Zener, Clarence Melvin, 1959 (3), College of Science, Texas A \& M University, College Station, Texas 77843

Zimm, Bruno Hasbrouck, 1958 (5), Department of Chemistry, University of California, San Diego, La Jolla, California 92037

Zinn, Walter Henry, 1956 (4), Combustion Engineering, Inc., P. O. Box 500, Windsor, Connecticut 06095

Zirkle, Raymond Elliott, 1959 (8), Department of Biophysics, University of Chicago, 5640 Ellis Avenue, Chicago, Illinois 60637 
Zworykin, Vladimir Kosma, 1943 (4), RCA Laboratories, David Sarnoff Research Center, Princeton, New Jersey 08540

Zygmund, Antoni, 1960 (1), Department of Mathematics, University of Chicago, Chicago, Illinois 60637

Number of Members July 1, 1967: 768

\section{MEMBERS EMERITI}

Albright, Fuller, 1952 (10), 271 Goddard Avenue, Brookline, Massachusetts 02146 Babcock, Harold Delos, 1933 (2), 811 East Washington Boulevard, Pasadena, California 91104

Bartelmez, George William, 1949 (8), 224 Agnes Avenue, Missoula, Montana 59801

Coolidge, William David, 1925 (3), 1480 Lenox Road, Schenectady, New York 12308

Hartman, Carl Gottfried, 1937 (8), 606 Crescent Avenue, Apt. 5 E, Plainfield, New Jersey 07060

Lambert, Walter Davis, 1949 (13), Cosmos Club, 2121 Massachusetts Avenue, N. W., Washington, D. C., 20008

Schultz, Adolph Hans, 1939 (11), Anthropologisches Institut, Künstlergasse 15, Zurich, Switzerland

Tyzzer, Ernest Edward, 1942 (10), 484 Water Street, Wakefield, Massachusetts 01880

Vandiver, Harry Shultz, 1934 (1), Box 7881, University of Texas, Austin, Texas 78712

Number of Members Emeriti July 1, 1967: 9

\section{FOREIGN ASSOCIATES}

The number in parentheses following the year of election indicates association within the Sections of the National Academy of Sciences.

Adrian, of Cambridge, Edgar Douglas, Baron, 1941 (9), Trinity College, Cambridge, England

Alexandroff, Paul A., 1947 (1), Mathematical Institute of the Academy of Sciences of the U. S. S. R., Bolshaya Kalushskaya 19, Moscow, U. S. S. R.

Alfvén, Hannes O. G., 1966 (13), Division of Plasma Physics, Royal Institute of Technology, Stockholm 70, Sweden

Amaldi, Edoardo, 1962 (3), University of Rome, Piazzale delle Scienze, 5, Rome, Italy

Ambartsumian, Victor Amazaspovich, 1959 (2), Burakan Astronomical Observatory, Erevan, Armenia, U. S. S. R.

Andrewes, Sir Christopher Howard, 1964 (10), Overchalke, Coombe Bissett, Salisbury, Wiltshire, England

Baltzer, Fritz, 1967 (8), Zoological Institute, University of Berne, Berne, Switzerland 
Bartlett, Sir Frederic Charles, 1947 (12), 161 Huntingdon Road, Cambridge, England

Best, Charles Herbert, 1950 (9), Banting and Best Department of Medical Research, University of Toronto, Toronto, Canada

Blackett, Patrick Maynard Stuart, 1966 (3), The Royal Society, Burlington House, London, W. 1, England

Born, Max, 1955 (3), Marcard Strasse 4, Bad Pyrmont, Germany

Brachet, Jean, 1965 (8), Laboratoire de Morphologie Animale, Université Libre de Bruxelles, 1850, Chaussée de Wavre, Brussels 16, Belgium

Bragg, Sir William Lawrence, 1945 (3), The Royal Institution, 21 Albemarle Street, London, W. 1, England

de Broglie, Prince Louis, 1948 (3), 94 Rue Perronet, Neuilly-sur-Seine, France

Brun, Edmond Antoine, 1960 (4), University of Paris, 8-10, place du Commerce, Paris XV, France

Bullard, Sir Edward Crisp, 1959 (13), Madingley Rise, Madingley Road, Cambridge, England

Bullen, Keith Edward, 1961 (13), Department of Applied Mathematics, University of Sydney, Sydney, Australia

Burnet, Sir Macfarlane, 1954 (10), c/o Department of Microbiology, University of Melbourne, Parkville, N. 2, Victoria, Australia

Caso, Alfonso, 1943 (11), Avenida Central 234, Tlacopac, Villa Obregón, Mexico 20, D. F.

Chapman, Sydney, 1946 (13), High Altitude Observatory, Boulder, Colorado 80302 , U.S. A.

Clark, Sir Wilfrid Le Gros, 1963 (11), Department of Human Anatomy, Oxford University, South Parks Road, Oxford, England

Dale, Sir Henry Hallett, 1940 (9), The Wellcome Trust, 52 Queen Anne Street, London, W. 1, England

Dirac, Paul Adrien Maurice, 1949 (1), Department of Mathematics, St. John's College, Cambridge, England

Eccles, Sir John Carew, 1966 (9), Institute for Biomedical Research, American Medical Association Education and Research Foundation, 535 North Dearborn Street, Chicago, Illinois 60610, U. S. A.

Eigen, Manfred, 1966 (5), Max-Planck-Institut für Physikalische Chemie, Bunsenstrasse 10, 3400 Göttingen, Germany

Ephrussi, Boris, 1961 (7), Laboratoire de Génétique Physiologique, 91, Gif-surYvette, France

Florey, of Adelaide, Howard Walter, Baron, 1963 (10), The Queen's College, Oxford, England

von Frisch, Karl, 1951 (8), The Zoological Institute, University of Munich, Munich, Germany

Geijer, Per, 1958 (6), Agnevaegen 5, Djursholm I, Sweden

Glen, Robert, 1967 (15), Canada Department of Agriculture, Ottawa, Ontario, Canada

Gorter, Cornelis Jacobus, 1967 (3), Kamerlingh Onnes Laboratory, State University of Leiden, Nieuwsteeg 18, Leiden, The Netherlands 
Gustafsson, Carl Åke Torsten, 1967 (7), Department of Forest Genetics, Royal College of Forestry, Stockholm 50, Sweden

Harrison, James Merritt, 1965 (6), Department of Mines and Technical Surveys, 588 Booth Street, Ottawa 4, Ontario, Canada

Hawthorne, William Rede, 1965 (4), University Engineering Laboratory, University of Cambridge, Trumpington Street, Cambridge, England

Heisenberg, Werner, 1961 (3), Max Planck Institut für Physik und Astrophysik, Aumeisterstrasse 6, Munich 23, Germany

Hill, Archibald Vivian, 1941 (9), 11A Chaucer Road, Cambridge, England

Hinshelwood, Sir Cyril Norman, 1960 (5), Department of Chemistry (Room 41), Imperial College, Imperial Institute Road, London, S.W. 7, England

Hodge, Sir William Vallance Douglas, 1959 (1), The Master's Lodge, Pembroke College, Cambridge, England

Hopf, Heinz, 1957 (1), Swiss Federal Institute of Technology, Zurich, Switzerland Houssay, Bernardo Alberto, 1940 (9), Viamonte 2790, Buenos Aires, Argentina

Jeffreys, Sir Harold, 1945 (13), St. John's College, Cambridge, England

Kapitza, Peter Leonidovich, 1946 (3), S. I. Vavilov Institute of Physical Problems, Academy of Sciences of the U. S. S. R., Moscow, U. S. S. R.

Karrer, Paul, 1945 (5), Spyristeig 30, Zurich 7, Switzerland

Katchalski, Ephraim, 1966 (14), Department of Biophysics, Weizmann Institute of Science, Rehovot, Israel

Kihara, Hitoshi, 1958 (7), National Institute of Genetics, Misima, Japan

Kolmogorov, Andrej N., 1967 (1), Moscow State University, Moscow, U.S.S.R.

Konorski, Jerzy, 1963 (12), Department of Neurophysiology, Nencki Institute of Experimental Biology, 3, Pasteur Street, Warsaw 22, Poland

Krebs, Sir Hans Adolf, 1964 (14), Department of Biochemistry, Oxford University, South Parks Road, Oxford, England

Kuno, Hisashi, 1963 (6), Geological Institute, University of Tokyo, Tokyo, Japan

Landau, Lev Davidovich, 1960 (3), S. I. Vavilov Institute of Physical Problems, Academy of Sciences of the U. S. S. R., Moscow, U. S. S. R.

Leloir, Luis F., 1960 (14), Instituto de Investigaciones Bioquimicas, Obligado 2490, Buenos Aires, Argentina

Leray, Jean, 1965 (1), Collège de France, 11 Place Marcelin-Berthelot, Paris V, France

Levi Strauss, Claude, 1967 (11), Laboratoire d'Anthropologie Sociale, Collège de France, 11 Place Marcelin-Berthelot, Paris V, France

Lorenz, Konrad, 1966 (8), Max-Planck-Institut für Verhaltensphysiologie, 8131 Seewiesen, Seewiesen über Starnberg (Obb.), Germany

Lundegädh, Henrik Gunnar, 1964 (7), Research Laboratory in Plant Physiology, Penningby, Sweden

Lwoff, André, 1955 (9), Institut Pasteur, Paris XV, France

Lynen, Feodor, 1962 (14), Max-Planck-Institut für Zellchemie, Karlstrasse 23-25, Munich 2, Germany

Medawar, Peter Brian, 1965 (10), National Institute for Medical Research, Mill Hill 3666, London, N. W. 7, England 
Minnaert, Marcel Gilles Jozef, 1964 (2), Utrecht Observatory, Zonnenburg 2, Utrecht, The Netherlands

Mott, Sir Nevill Francis, 1957 (3), University of Cambridge, Cavendish Laboratory, Free School Lane, Cambridge, England

Oort, Jan Hendrik, 1953 (2), Observatory of Leiden, Leiden, The Netherlands

Penfield, Wilder, 1953 (10), Montreal Neurological Institute, 3801 University Street, Montreal 2, Canada

Penney, Sir William George, 1962 (4), United Kingdom Atomic Energy Authority, Charles II Street, London, S. W. 1, England

Piaget, Jean, 1966 (12), Institut des Sciences de l'Éducation, Université de Genève, Palais Wilson, Geneva, Switzerland

Prelog, Vladimir, 1961 (5), Laboratorium für organische Chemie, Eidgenossische Technische Hochschule, Zurich, Switzerland

Prigogine, Ilya, 1967 (5), Université Libre de Bruxelles, Avenue F-D. Roosevelt, 50 , Brussels 5, Belgium

Reichstein, Tadeus, 1952 (5), Organisch-chemische Anstalt, St. Johanns-Ring 19, Basel, Switzerland

Robertson, Rutherford Ness, 1962 (7), Department of Botany, University of Adelaide, Adelaide, South Australia

Robinson, Sir Robert, 1934 (5), Shell Research Ltd., Shell Centre, Downstream Building, London, S. E. 1, England

Roy, Maurice, 1964 (4), 86, Avenue Niel, Paris XVII, France

Ruzicka, Leopold, 1944 (5), Department of Organic Chemistry, Institute of Technology, Zurich, Switzerland

Sander, Bruno Hermann Max, 1966 (6), Mineralogisches Institut, Alte Universität, Universitätstrasse 4, 6020 Innsbruck, Austria

Sanger, Frederick, 1967 (14), Medical Research Council, Laboratory of Molecular Biology, University Postgraduate Medical School, Hills Road, Cambridge, England

Semenov, Nikolai Nikolaevich, 1963 (5), Institute of Chemical Physics, Vorobyevskoye chaussee 2, Moscow, V-133, U. S. S. R.

Southwell, Sir Richard Vynne, 1943 (4), The Old House, Trumpington, Cambridge, England

Svedberg, The, 1945 (5), Fysikalisk-Kemiska Institutionen, Uppsala University, Uppsala, Sweden

Swings, Pol, 1966 (2), Institut d'Astrophysique, Université de Liège, CointeSclessin, Belgium

Tamiya, Hiroshi, 1966 (7), The Tokugawa Institute for Biological Research, Toshima-ku, Mejiro, Tokyo, Japan

Taylor, Sir Geoffrey Ingram, 1945 (1), Trinity College, Cambridge, England

Theorell, Axel Hugo, 1957 (14), Nobel Institute of Medicine, Solnavagen 1, Stockholm 60, Sweden

Tilley, Cecil Edgar, 1967 (6), Department of Mineralogy and Petrology, Cambridge University, Downing Place, Cambridge, England

Tiselius, Arne W. K., 1949 (14), Institute of Biochemistry, Uppsala University, Uppsala, Sweden 
Todd, of Trumpington, Alexander Robertus, Baron, 1955 (5), University of Cambridge, University Chemical Laboratory, Lensfield Road, Cambridge, England

Tomonaga, Sin-itiro, 1965 (3), Science Council of Japan, Ueno Park, Tokyo, Japan

Wagner, Carl, 1967 (4), Max-Planck-Institut für Physikalische Chemie, Bunsenstrasse 10, 34 Göttingen, Germany

Watson, D. M. S., 1938 (8), University College, Gower Street, London, W. C. 1, England

Yukawa, Hideki, 1949 (3), Yukawa Hall, Kyoto University, Kyoto, Japan

Number of Foreign Associates July 1, 1967: 87 